\title{
VLT-CRIRES SURVEY OF ROVIBRATIONAL CO EMISSION FROM PROTOPLANETARY DISKS
}

\author{
J. M. Brown ${ }^{1,2}$, K. M. Pontoppidan ${ }^{3}$, E. F. van Dishoeck ${ }^{2,4}$, G. J. Herczeg ${ }^{5}$, G. A. Blake ${ }^{6}$ And A. Smette $^{7}$ \\ ${ }^{1}$ Harvard-Smithsonian Center for Astrophysics, 60 Garden Street, MS 78, Cambridge, MA 02138, USA; joannabrown@cfa.harvard.edu \\ ${ }^{2}$ Max-Planck-Institut für extraterrestrische Physik, Postfach 1312, D-85741 Garching, Germany \\ ${ }^{3}$ Space Telescope Science Institute, 3700 San Martin Drive, Baltimore, MD 21218, USA \\ ${ }^{4}$ Leiden Observatory, Leiden University, P.O. Box 9513, NL-2300 RA Leiden, The Netherlands \\ ${ }^{5}$ The Kavli Institute for Astronomy and Astrophysics, Peking University, Yi He Yuan Lu 5, Hai Dian Qu, Beijing 100871, China \\ ${ }^{6}$ Division of Geological \& Planetary Sciences, California Institute of Technology, Pasadena, CA 91125, USA \\ ${ }^{7}$ ESO, Alonso de Cordova 3107, Casilla 19001, Vitacura, Chile \\ Received 2012 October 4; accepted 2013 April 15; published 2013 May 29
}

\begin{abstract}
We present a large, comprehensive survey of rovibrational CO line emission at $4.7 \mu \mathrm{m}$ from 69 protoplanetary disks, obtained with CRIRES on the ESO Very Large Telescope at the highest available spectral resolving power $\left(R=95,000, \Delta v=3.2 \mathrm{~km} \mathrm{~s}^{-1}\right)$. The CO fundamental band $(\Delta v=1)$ is a well-known tracer of warm gas in the inner, planet-forming regions of gas-rich disks around young stars, with the lines formed in the super-heated surfaces of the disks at radii of 0.1-10 AU. Consistent with earlier studies, the presence of 100-1000 K CO is found to be ubiquitous around young stars which still retain disks. Our high spectral resolution data provide new insight into the kinematics of the inner disk gas. The observed line profiles are complex and reveal several different components. Pure double-peaked Keplerian profiles are surprisingly uncommon in our sample, beyond the frequency expected based on disk inclination. The majority of the profiles are consistent with emission from a disk plus a slow (few $\mathrm{km} \mathrm{s}^{-1}$ ) molecular disk wind. This is evidenced by analysis of different classes as well as an overall tendency for line profiles to have excess emission on their blue side. The data support the notion that thermal molecular winds are common for young disks. Thanks to the high spectral resolution, narrow absorption lines and weak emission lines from isotopologues and from vibrationally excited levels are readily detected. In general, ${ }^{13} \mathrm{CO}$ lines trace cooler gas than the bulk ${ }^{12} \mathrm{CO}$ emission and may arise from further out in the disk, as indicated by narrower line profiles. A high fraction of the sources show vibrationally excited emission $(\sim 50 \%)$ which is correlated with accretion luminosity, consistent with ultraviolet fluorescent excitation. Disks around early-type Herbig AeBe stars have narrower line profiles, on average, than their lower-mass late-type counterparts, due to their increased luminosity. Evolutionary changes in $\mathrm{CO}$ are also seen. Removal of the protostellar envelope between class I and II results in the disappearance of the strong absorption lines and $\mathrm{CO}$ ice feature characteristic of class I spectra. However, $\mathrm{CO}$ emission from class I and II objects are similar in detection frequency, excitation, and line shape, indicating that inner disk characteristics are established early.
\end{abstract}

Key words: infrared: general - protoplanetary disks - stars: formation - stars: pre-main sequence - stars: protostars

Online-only material: color figures, extended figure

\section{INTRODUCTION}

The inner regions of gas-rich protoplanetary disks $(R \lesssim$ $10 \mathrm{AU})$ are thought to be the birthplaces of most giant planets (Armitage 2010; Kley \& Nelson 2012). The chemical and physical processes sculpting these environments in the first few million years of the life of a star are critical for determining many properties of mature planetary systems. These include the formation of rocky "oligarchs"- the building blocks of terrestrial planets (Nagasawa et al. 2007) - and comets and water-rich asteroids important for the delivery of water and organics to planetary surfaces (Raymond et al. 2004). The interaction between the gas-rich inner disk and protoplanets has the power to rearrange the orbital structure of the entire planetary system (Armitage 2011), allowing for significant modification of radial chemical abundance structures; for instance, the chemical boundary defined by the snow-line may not always be predictive for the compositions of planets in mature systems.

A key diagnostic of the structure of planet-forming regions is the fundamental $(\Delta v=1)$ rovibrational band of $\mathrm{CO}$ at $4.7 \mu \mathrm{m}$. It is particularly sensitive to gas temperatures of $100-1000 \mathrm{~K}$, corresponding to radii of 0.1-10 AU in typical protoplanetary disks around solar-mass pre-main sequence stars. Because of the high opacity of dust at $5 \mu \mathrm{m}$ and the low temperatures of disk midplanes beyond $\sim 1 \mathrm{AU}$, the $\mathrm{CO}$ fundamental band typically traces the disk at high altitude, specifically the so-called "warm molecular layer" (Aikawa et al. 2002; Gorti \& Hollenbach 2008; Woitke et al. 2009). In comparison with the total disk surface densities in the planet-forming region of $\Sigma=10-1000 \mathrm{~g} \mathrm{~cm}^{-2}$, the CO fundamental band traces roughly $N_{\mathrm{H}} \sim 10^{21}-10^{23} \mathrm{~cm}^{-2}$, corresponding to $10^{-3}$ to $10^{-1} \mathrm{~g} \mathrm{~cm}^{-2}$, where $N_{\mathrm{H}}$ is the total column of hydrogen nuclei.

While the warm molecular layer represents a small fraction of the total vertical disk column, its structure is intimately linked to key properties of the bulk gas in protoplanetary disks, relevant to their evolution and ability to form planets. Through high temperatures and interactions with ionizing stellar radiation, the molecular layer acts as a chemical factory, producing water and complex organics (Markwick et al. 2002; Glassgold et al. 2009; Woods \& Willacy 2009; Walsh et al. 2012). It also forms a boundary between the deep, neutral, and inactive disk midplane and the uppermost ionized layers. As such, the physical and kinematical structure of the molecular layer traces thermal and photo-evaporative flows from the disk surface, controlling outward radial mixing and mass loss (Hollenbach et al. 1994; Owen et al. 2010). It is in the molecular layer that the stellar 
magnetic field can couple to the disk and drive turbulence and accretion flows (Gammie 1996; Perez-Becker \& Chiang 2011). Finally, the molecular layer responds to dynamical perturbations caused by the presence of giant protoplanets, leading to potentially observable effects (Regály et al. 2010).

Rovibrational $\mathrm{CO}$ emission is present throughout a wide range of protoplanetary disks from still embedded protostars (Pontoppidan et al. 2003) to transitional disks with inner dust holes (Salyk et al. 2009), and from low mass T Tauri stars (Najita et al. 2003) to higher mass Herbig stars (Brittain et al. 2007). The CO lines are formed by a combination of collisional excitation, infrared (vibrational) pumping, and ultraviolet (UV; electronic) fluorescence. The relative importance of these excitation processes depends on location within the disk and the shape and strength of the radiation field from the central star (Blake \& Boogert 2004).

The advent of the CRyogenic high-resolution InfraRed Echelle Spectrograph (CRIRES) instrument on the Very Large Telescope (VLT) has opened the possibility of observing the $\mathrm{CO}$ fundamental bands at higher spectral resolving power $(R \approx 95,000)$ and higher spatial resolution than before, thus providing new insight into this critical planet-forming region of the disk. We present here the results of a large VLT-CRIRES program of 69 disks around low- and intermediate-mass stars and 22 embedded young stellar objects (Pontoppidan et al. 2011b). Previous papers have used subsets of our CRIRES database to address a variety of questions. Bast et al. (2011) investigate the presence and origin of a class of broad single-peaked CO rovibrational line profiles indicating non-Keplerian gas motions. Using spectro-astrometry, Pontoppidan et al. (2008, 2011a) constrain the structure of the gas emission and velocity fields on milli-arcsecond scales in a smaller sample of disks. Some disks show CO emission consistent with simple Keplerian models, whereas other disks, especially those in the Bast et al. (2011) sample, show a spectro-astrometry pattern consistent with a slow molecular disk wind. For transitional disks with a large inner dust hole or gap, Pontoppidan et al. (2008) pinpointed the origin of $\mathrm{CO}$ rovibrational emission from inside the dust gap whereas Brown et al. (2012) resolved the $\mathrm{CO}$ emission near the outer wall of the hole around the Herbig star Oph IRS 48. Herczeg et al. (2011) characterized the progenitors to protoplanetary disks, when the sources are still embedded in protostellar envelopes. Thi et al. (2010) found evidence for episodic outflow activity with winds up to $100 \mathrm{~km} \mathrm{~s}^{-1}$ in broad blueshifted lines toward one object, whereas Herczeg et al. (2011) found the same phenomenon for a handful of other embedded sources.

Finally, the high spectral resolution of CRIRES also boosts the line to continuum ratio and thus allows the detection of weak lines from minor species. Smith et al. (2009) accurately measured the isotopologue ratios of the four major $\mathrm{CO}$ species $-{ }^{12} \mathrm{CO},{ }^{13} \mathrm{CO}, \mathrm{C}^{18} \mathrm{O}$, and $\mathrm{C}^{17} \mathrm{O}$-in the circumstellar environment of two young stars. The aim was to search for mass-independent oxygen isotope fractionation relative to the interstellar medium in order to understand the solar system oxygen anomaly. Mandell et al. (2012) searched for near-infrared lines of small organic molecules $\left(\mathrm{HCN}, \mathrm{C}_{2} \mathrm{H}_{2}, \mathrm{CH}_{4}\right)$ in a few sources. While these studies discuss the detailed properties of specific sub-samples, this paper provides an overview of the basic properties of $\mathrm{CO}$ rovibrational emission from protoplanetary disks around young solar-type stars at the highest available spectral resolution.

Using the entire CRIRES data set, we aim to address the following questions related to the structure and evolution of planet-forming regions of protoplanetary disks. (1) Where is the $\mathrm{CO}$ gas located? What fraction of sources show emission from a radially flowing surface such as a slow disk wind? (2) What are typical temperatures probed by $\mathrm{CO}$ and its isotopologues and how do these values compare with current thermo-chemical models of the inner disk? How are the $\mathrm{CO}$ fundamental bands excited, and what consequences does the excitation mechanism have for the lines as a tracer of inner disk surfaces? (3) Do CO line profiles depend on stellar spectral type, and is this a tracer of the prevalence of UV fluorescent excitation over thermal excitation? (4) What evolutionary changes occur in the gas?

\section{OBSERVATIONS AND SAMPLE CHARACTERISTICS}

\subsection{The CRIRES Survey}

In this paper, we analyze high resolution spectra of the 4.7 micron $\mathrm{CO} v=1-0$ fundamental emission band using the CRIRES on the VLT of the European Southern Observatory (ESO; Kaeufl et al. 2004). CRIRES operates at high resolution $\left(R=95,000, \Delta v=3.2 \mathrm{~km} \mathrm{~s}^{-1}\right)$ using a 0.2 slit. It is fed by a Multi-Application Curvature Adaptive Optics (MACAO) system, which allows correction of atmospheric turbulence and can provide diffraction limited images at the focal plane, therefore improving the overall instrument sensitivity.

The sample consists of 91 young stars-69 protoplanetary disks around young stars and 22 embedded protostars that are still surrounded by massive remnant envelopes. The data were obtained as part of an ESO Large Programme 8 to study infrared molecular emission from solar-type protostars and protoplanetary disks (Pontoppidan et al. 2011b). ${ }^{9}$ We discuss here primarily the disk sample, but compare our results with those of the embedded protostars presented in Herczeg et al. (2011) to search for evolutionary trends between embedded and classical protoplanetary disks.

\subsection{Sample Selection}

The disk sample spans a range of physical properties including spectral type, stellar mass and luminosity, and inclination. Such a large sample of $\mathrm{CO}$ emission profiles provides an opportunity for a broad examination of the gas distribution in a wide variety of circumstellar environments. The sample was chosen to include protoplanetary disks around solar-mass $\left(0.5-2.0 M_{\odot}\right)$ young stars (see Figure 1). A range of evolutionary states were sampled from massive gas-rich disks that are optically thick to visible and UV radiation to transition disks with inner dust holes or gaps. The sources are located in the nearby star forming regions visible from Paranal Observatory, including Taurus, Ophiuchus, Serpens, Corona Australis, and Chamaeleon. All these regions have undergone recent active star formation and have ages of approximately 1-5 Myr (Greene \& Meyer 1995; Armitage et al. 2003; Oliveira et al. 2009). Protoplanetary disks were selected based on brightness ( $\gtrsim 100 \mathrm{mJy}$ at $4.7 \mu \mathrm{m})$, as well as the existence of prior data sets characterizing the structure of the disks and supporting the presence of significant gas reservoirs. The prior data sets defining the sample include Spitzer spectroscopy from the cores to disks (c2d) Spitzer Legacy survey (Evans et al. 2003), the Keck-NIRSPEC 3-5 $\mu \mathrm{m}$ protoplanetary disk survey (e.g., Blake \& Boogert 2004; Salyk et al. 2011), and the VLT-ISAAC protostellar survey (van Dishoeck

\footnotetext{
8 This work is based on observations collected at the European Southern Observatory Very Large Telescope under program ID 179.C-0151.

9 Fully processed spectra are available at http://www.stsci.edu/ $\sim$ pontoppi.
} 


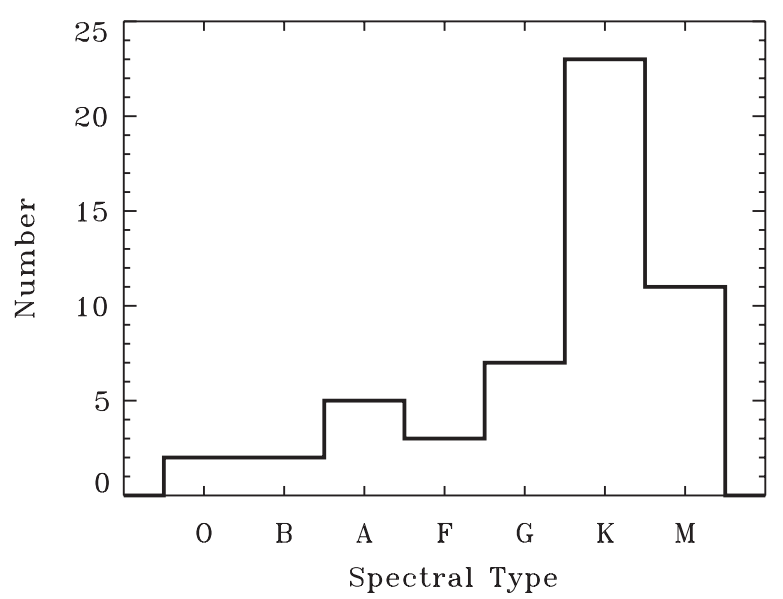

Figure 1. Histogram of the spectral types of the stars in our disk sample. The sample is dominated by $\mathrm{K}$ type stars. When a range of spectral types were reported in the literature the mean value was taken.

et al. 2003). We limited the survey to disks around solar-type stars $\left(0.1 M_{\odot} \lesssim M_{*} \lesssim 2.0 M_{\odot}\right)$, excluding most Herbig AeBe stars-the focus of complementary CRIRES surveys (e.g., van der Plas et al. 2009).

\subsection{Observing Procedure and Data Reduction}

The high spectral resolution and high dynamic range of CRIRES spectra, a factor of four improvement in spectral resolution over most previous $\mathrm{CO}$ surveys, fully resolves the individual line profiles so that velocity information can be used to locate the gas within the disk. The resolution is particularly needed in the cases of disks with low inclinations where the lines are intrinsically narrow and in the cases where multiple components, particularly absorption features, contribute to the line profile. Because of the adaptive optics system, line emission can be spatially resolved down to angular scales of $\sim 0^{\prime \prime} .1$.

The observations were taken between 2007 April and 2010 March. Table 1 lists the targets observed and the dates and wavelength settings of the observations. Multiple spectral settings were taken to cover a range of rotational $J$ lines. Each spectral setting is observed with four different detectors, leading to four discontinuous wavelength regions. The wavelength listed is the center of the third detector and the range is -70 to $+39 \mathrm{~nm}$ on either side of the listed wavelength, with $\sim 6 \mathrm{~nm}$ gaps between the detectors. The spectra cover the CO $P$ branch lines with $\Delta J=-1$ and a few low energy lines from the $R$ branch $(\Delta J=1) .{ }^{12} \mathrm{CO} v=1-0$ lines are the most prominent. Gas phase lines from higher vibrational states and rarer $\mathrm{CO}$ isotopologues such as ${ }^{13} \mathrm{CO}, \mathrm{C}^{18} \mathrm{O}$, and $\mathrm{C}^{17} \mathrm{O}$ are also included in the wavelength settings.

The spectra were obtained using an ABBA 10" nodding pattern permitting a first order correction of the infrared background by pair subtraction. Integration times were generally 8-16 minutes per spectral setting. The reduction of the spectra is described in detail in Pontoppidan et al. (2008, 2011a). Wavelength calibration used the prevalent telluric features in the standard star spectra. Atmospheric features were removed by dividing the source spectrum by the standard star spectrum. Standard stars were observed close in time and elevation to minimize atmospheric differences. Remaining small discrepancies from airmass differences and subpixel variations in wavelength solution were corrected by scaling or shifting the standard spectrum to minimize telluric residuals. Remaining strong telluric

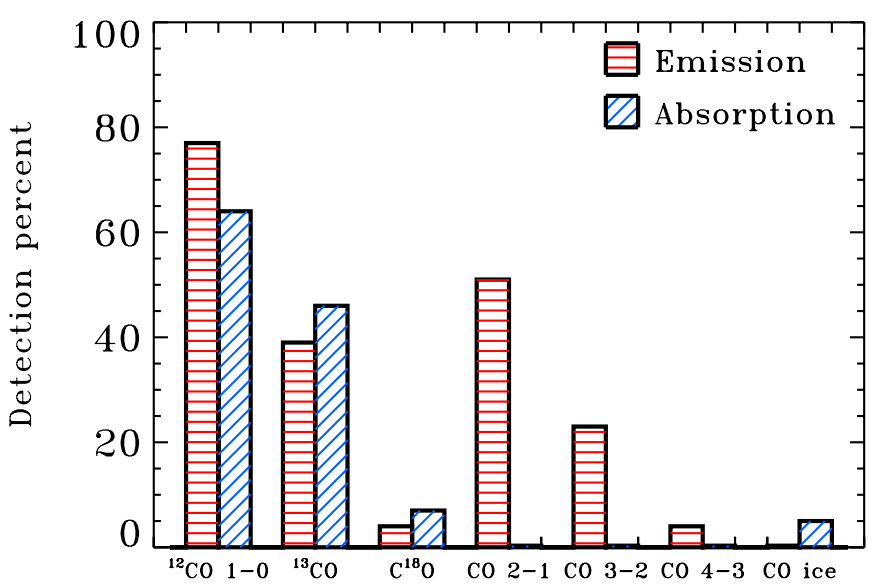

Line

Figure 2. Percentage of disk sources with different lines detected in emission (red, horizontally hashed) and absorption (blue, diagonally hashed). In general, rarer isotopologues and higher energy lines are less frequently detected. The CO isotopologues are more commonly seen in absorption while the vibrationally excited transitions are seen exclusively in emission. Table 2 lists common lines detected for individual sources.

(A color version of this figure is available in the online journal.)

features were blanked from the analyzed spectra. The observations were preferentially scheduled for periods when the Earth's velocity around the Sun created large shifts of the source lines relative to the telluric features. When possible, complete wavelength coverage was obtained using the Earth's orbital motion between separated observation dates to Doppler shift the telluric features relative to the source lines. The spectra from two or more dates were then combined, barring large differences in line profiles.

The accuracy of the absolute flux calibration is $\sim 30 \%$, estimated by comparing raw counts of consecutive nod pairs. This flux calibration is limited by differences in Strehl ratio between the target disk and the standard star, as well as by pointing jitter. We therefore scaled the spectra to known $M$ band fluxes (see Table 2), with many from the Spitzer IRAC 2 band. Note, however, that some protoplanetary disks are known to be variable on short time scales at the $10 \%-60 \%$ level (Flaherty et al. 2012) and discrepancies could exist based on the different aperture sizes. In cases where no Spitzer photometry was available a linear relationship between the maximum counts received and the flux was determined based on the sources with known fluxes. Determining the flux using this method led to a median error in derived fluxes of $\sim 20 \%$ for sources with previously known fluxes. However, errors were as large as a factor of two in exceptional cases. The determination was more accurate with larger numbers of exposures.

\subsection{Detection Rates}

$\mathrm{CO}$ gas phase lines are common in our sample, both in emission and in absorption, as shown graphically in Figure 2. Lines are commonly a combination of emission and absorption resulting from two distinct gas components such as disk emission with foreground absorption. Overall, emission features are seen from $53 / 69(77 \%)$ of the disks. Absorption is seen in 44/69 $(64 \%)$ of the spectra. CO $v=2-1$ lines are seen from 36/69 $(52 \%)$ of the sample, always in emission. ${ }^{13} \mathrm{CO}$ emission lines are seen in $27(39 \%)$ of the sources. However, ${ }^{13} \mathrm{CO}$ is more commonly seen in absorption $(46 \%)$, often at cold temperatures 
Table 1

Summary of CRIRES Disk Observations

\begin{tabular}{|c|c|c|}
\hline Source & $\begin{array}{l}\text { Wavelength }^{\mathrm{a}} \\
(\mathrm{nm})\end{array}$ & Date of Observation $^{\mathrm{a}}$ \\
\hline AA Tau & $4616,4730,4868$ & 2007 Oct 12 \\
\hline AS $205 \mathrm{~S}$ & $4662.1,4676.1,4760.8,4773.6$ & 2007 Apr 21 \\
\hline AS 209 & 4716,4730 & 2008 May 1 \\
\hline CRBR 2422.8-3423 & 4716,$4730 ; 4868$ & 2008 Aug 3; 2008 Aug 4 \\
\hline CW Tau & $4716,4868,4946.2 ; 4730$ & 2008 Dec 30; 2009 Jan 1 \\
\hline DF Tau & 4716,4730 & 2009 Jan 1 \\
\hline DG CrA & $4716,4730,4833 ; 4946.2$ & 2007 Apr 25; 2008 Aug 9 \\
\hline DG Tau & $4716,4730,4868$ & 2007 Oct 15 \\
\hline DoAr 21 & 4716,4730 & 2008 Apr 30 \\
\hline DoAr 24E S (B) & 4716,$4730 ; 4730$ & 2007 Sep 2; 2007 Sep 3 \\
\hline DoAr 24E N (A) & 4716,4730 & 2007 Sep 2 \\
\hline DR Tau & $4716,4730,4833 ; 4716,4946.2$ & 2007 Oct $10 ; 2008$ Dec 30 \\
\hline EC 82 & 4716,4730 & 2007 Apr 22 \\
\hline Elias 23 & $4716,4730,4868 ; 4946$ & 2008 Aug 06; 2010 Mar 4 \\
\hline EX Lup & 4716,$4730 ; 4716,4730,4868$ & 2008 Apr 27; 2008 Aug 6 \\
\hline FN Tau & $4868 ; 4730$ & 2007 Oct 16; 2008 Dec 29 \\
\hline FZ Tau & 4716 & 2009 Jan 1 \\
\hline GQ Lup & $4662.1,4676.1,4760.8,4773.6 ; 4730$ & 2007 Apr 21; 2008 May 2, 2008 Aug 4 \\
\hline HBC 680 & 4716,4730 & 2007 Sep 1 \\
\hline HD $135344 \mathrm{~B}$ & $4716,4730,4929.3,4946.2$ & 2007 Apr 22 \\
\hline HD 142527 & $4716,4730,4868 ; 4710 ; 4076,4101$ & 2008 Aug 5; 2008 Aug 7; Aug 9 \\
\hline HD 144432 S \& N* & 4710,4730 & 2008 Aug 2 \\
\hline HD 144965 A $(\mathrm{N}) \& \mathrm{~B}^{*}$ & 4716,4730 & 2008 Apr 29 \\
\hline HD 176386 & 4730 & 2007 Sep 1 \\
\hline Haro 1-4 A (S) \& B* & $4716,4730,4833$ & 2008 Apr 29 \\
\hline IM Lup & $4716,4730,4833$ & 2008 Apr 26 \\
\hline IRS 48 & $4730 ; 4730,4833 ; 4710,4730 ; 4868$ & 2007 Sep 5; 2008 May 3; Aug 2; Aug 5 \\
\hline IRS 51 & 4716,4730 & 2008 Aug 3 \\
\hline LkHa 330 & $4730 ; 4868 ; 4716,4730$ & 2007 Oct $10 ;$ Oct 12; 2008 Dec 29 \\
\hline $\mathrm{R}$ CrA & $4760.8 ; 4730 ; 4946.2$ & 2007 Apr 21; Sep 1; 2008 Aug 9 \\
\hline RNO 90 & $4716,4730,4833,4929.3 ; 4730$ & 2007 Apr 25; Apr 26 \\
\hline RR Tau & 4730,4868 & 2007 Oct 15 \\
\hline RU Lup & $4716,4730,4833,4929.3 ; 4730$ & 2007 Apr 26; 2008 Apr 27 \\
\hline RW Aur & 4868 & 2007 Oct 15 \\
\hline RY Lup & $4716,4730,4840,4929.3 ; 4716,4730,4833$ & 2007 Apr 24; 2008 Apr 26 \\
\hline S CrA N \& S & 4716,$4730 ; 4730 ; 4946.2$ & 2007 Apr 22; 2007 Sep 3; 2008 Aug 9 \\
\hline Serp $32^{b}$ & 4716,4730 & 2007 Sep 2 \\
\hline Serp $64^{c}$ & 4716 & 2007 Aug 30 \\
\hline SO 411 & 4868 & 2007 Oct 14 , Oct 16 \\
\hline SR 9 A $(S) \& B^{*}$ & 4716 & 2008 Aug 8 \\
\hline SR 21 & 4716,$4730 ; 4730,4833$ & 2007 Aug 30; Aug 31 \\
\hline SR $24 \mathrm{~A}(\mathrm{~S}) \& \mathrm{~B} / \mathrm{C}(\mathrm{N})$ & $4716,4730,4833$ & 2008 May 3 \\
\hline SX Cha A \& B* & $4800,4946.2$ & 2008 Dec 30 \\
\hline SY Cha & $4800,4946.2$ & 2009 Jan 3 \\
\hline Sz 68 A,B \& C & $4716,4730,4833,4929.3$ & 2007 Apr 26 \\
\hline T Cha & 4800,$4820 ; 4946.2$ & 2008 Dec 29; Dec 30 \\
\hline $\mathrm{T}$ CrA & 4716,$4730 ; 4730,4833,4929.3$ & 2007 Apr 23; Apr 26 \\
\hline T Tau N \& S (A) & 4730 & 2007 Oct 10 \\
\hline TW Cha & $4800,4820,4946.2$ & 2009 Jan 2 \\
\hline TW Hya & $4662.1,4760.8,4773.6 ; 4730$ & 2007 Apr 21; Apr 26 \\
\hline TY CrA & 4716,4730 & 2007 Apr 24 \\
\hline UX Tau & 4730,4868 & 2007 Oct 12 \\
\hline VSSG 1 & $4716,4730,4868$ & 2008 Aug 6 \\
\hline VV CrA N \& S & $4716,4730,4840 ; 4770,4779.5 ; 4946.2$ & 2007 Apr 24; Aug 31; 2008 Aug 9 \\
\hline VV Ser & $4662.1,4676.1,4760.8,4773.6 ; 4730$ & 2007 Apr 21; 2008 May 1 \\
\hline
\end{tabular}


Table 1

(Continued)

\begin{tabular}{lcc}
\hline \hline Source & $\begin{array}{c}\text { Wavelength }^{\mathrm{a}} \\
(\mathrm{nm})\end{array}$ & Date of Observation $^{\mathrm{a}}$ \\
\hline WL 22 & 4716,4730 & 2008 Aug 3 \\
WX Cha & $4946.2 ; 4830$ & 2008 Dec 30; 2009 Jan 2
\end{tabular}

Notes. This table summarizes the disk sources. See Appendix C, Table 8 for the complete embedded source list (also Herczeg et al. 2011 for low mass embedded sources).

${ }^{a}$ Semicolons (;) divide different groups of observations (e.g., for VW Cha A, the 4800 and 4820 settings were both observed on December 29 while the 4946.2 setting was observed on December 30).

${ }^{\text {b }}$ Serp 32 is a Spitzer selected source at 18:28:45.6 -00:07:21.6, and is probably a background giant/AGB star based on the high radial velocity. The source is not counted in the disk statistics.

${ }^{\mathrm{c}}$ Serp 64 is a Spitzer selected source at 18:29:01.7 +00:29:38.7.

* Detected companion was too faint for further analysis.

indicative of foreground material. $\mathrm{C}^{18} \mathrm{O}$ is seen in emission from four sources (6\%) and in absorption from seven sources (10\%).

\subsection{Multiplicity}

In cases where a binary companion was known, we oriented the slit to observe both stars. There are eight disk systems where both components are well separated and bright enough for analysis: AS 205, DoAr 24 E, S CrA, SR 24, SR 9, Sz 68, T Tau, and VV CrA. Six additional disk systems have detectable companions but the $M$-band secondary is too faint for any further analysis: Haro 1-4, HD 144432S, HD 144965, SR 9, SX Cha, and VW Cha. In some cases, the two components are blended in our data. HBC 680 consists of a pair of similar luminosity stars with a separation of 0 '.22 (Köhler et al. 2008). The northern component of SR 24 is a blend of the B and C components with a separation on 0'081 (Correia et al. 2006).

The spectra from the two components of a binary can look very different. AS 205, DoAr 24 E, T Tau, and VV CrA all have one component seen in emission and the other in absorption. In the case of $\mathrm{T}$ Tau, the northern component is viewed face on while the southern component is seen through its disk (Walter et al. 2003). In other cases, one star may be seen through the disk of the other (Smith et al. 2009).

\subsection{Extended Emission}

Good AO correction allows spatially extended sources to be seen directly in the CRIRES sample without using spectroastrometry. Brown et al. (2012) present a very extended ring in IRS 48, whereas Herczeg et al. (2011) examine extended emission in two embedded sources. Extended emission is not common in our sample, however. Only four additional sources have directly imaged extended emission: EC 82, LLN 19, R CrA, and T CrA (see Appendix A). Limits for the remaining pointlike objects are generally around 3 to $4 \mathrm{AU}$ (see also Bast et al. 2011).

\subsection{Temporal Variability}

Young stars are variable on relatively short timescales in a variety of diagnostics from optical fluxes, accretion line diagnostics, and even at longer mid-IR fluxes (Flaherty et al. 2012; Muzerolle et al. 2009). Two or more epochs in the same setting were obtained for 11 sources. We approach the search for variability in two ways: first, by simply comparing the line fluxes from the two observations and searching for significant systematic differences (taking into account our large error bars),

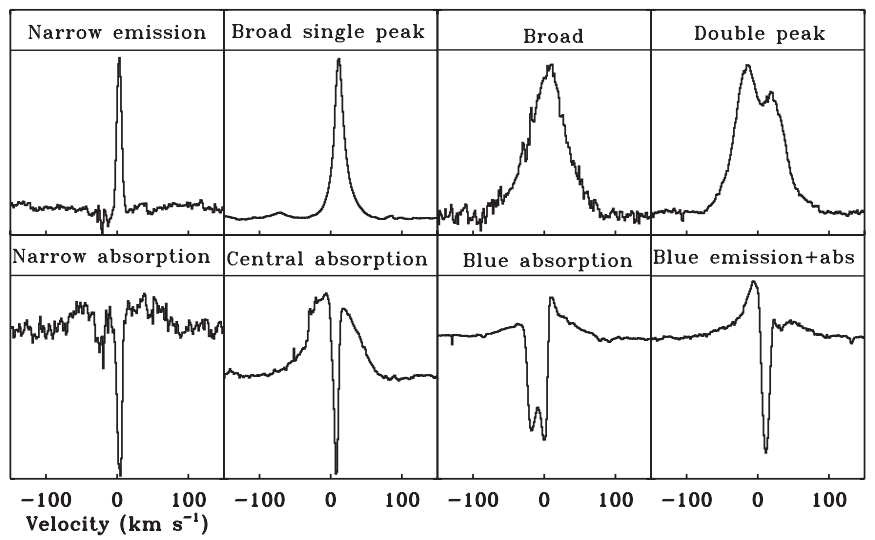

Figure 3. Gallery of the eight morphological line profile types discussed in Section 3 . The profiles are calculated by averaging observed ${ }^{12} \mathrm{CO} v=1-0$ lines between $R(10)$ and $P(32)$, avoiding blended lines.

and second, by closely examining the line profiles for differences in shape.

Overall, we find on timescales of up to two years a perhaps surprising lack of variability in most of the line profiles. A clear exception is EX Lup, which was undergoing a large outburst during the period of our program (see Goto et al. 2011 for further details). RU Lup shows a systematic decrease in line flux by $60 \%$ between 2007 April and 2008 April. The decrease could be due to either an increase in the continuum level or a decrease in the CO flux. Another example is a disappearance of the VV CrA outflow absorption from 2007 April to August. Further details on variability will be available in K. M. Pontoppidan et al. (2013, in preparation).

\section{CLASSIFICATION OF CO LINE PROFILES}

Figure 3 shows representative examples of the different $\mathrm{CO}$ line profiles of the disk sources. The profiles are often shaped by both emission and absorption components. In order to estimate the relative importance of the various physical components contributing to the observed $\mathrm{CO}$ lines, we divide the sources into groups based on the line profiles. The classification is summarized in Table 3. We discuss physical interpretations of these different categories in Section 5. Figure 4 shows a close up of the $P(8)$ line at $4.7359 \mu \mathrm{m}$ for all sources. In most cases we do not find any significant dependence of the emission line shape with rotational quantum number within the observed range (typically $R(1)-P(32)$ ). S CrA B and DG CrA are exceptions with a colder narrow component and a broader warm component in the ${ }^{12} \mathrm{CO}$ profile (see also Bast et al. 2011). 
Table 2

Summary of Observed Line Morphology

\begin{tabular}{|c|c|c|c|c|c|}
\hline Source & $\begin{array}{c}M \text { Flux } \\
(\mathrm{Jy})\end{array}$ & $\begin{array}{c}P(8) \text { Flux } \\
\left(\mathrm{Jy} \mathrm{km} \mathrm{s}^{-1}\right)\end{array}$ & ${ }^{12} \mathrm{CO}$ & ${ }^{13} \mathrm{CO}$ & ${ }^{12} \mathrm{CO} 2-1$ \\
\hline AA Tau & $0.3^{1}$ & 11.0 & $\mathrm{E} / \mathrm{A}$ & $\mathrm{E}$ & $\mathrm{E}$ \\
\hline AS $205 \mathrm{~N}$ & $4.3^{2}$ & 112 & E & $\mathrm{E}$ & $\mathrm{E}$ \\
\hline AS $205 \mathrm{~S}$ & $5.4^{2}$ & $16.8 /-59.7$ & $\mathrm{E} / \mathrm{A}$ & A & \\
\hline AS 209 & $1.4^{3}$ & 15.2 & $\mathrm{E} / \mathrm{FA}$ & & \\
\hline CRBR 2422.8-3423 & $0.4^{3}$ & -0.6 & A & A & \\
\hline CV Cha & $1.4^{3}$ & 32.9 & $\mathrm{E} / \mathrm{FA}$ & $\mathrm{E} / \mathrm{FA}$ & $\mathrm{E}$ \\
\hline CW Tau & $1.6^{4}$ & $44.9 /-14.3$ & $\mathrm{E} / \mathrm{A}$ & $\mathrm{E} / \mathrm{A}$ & $\mathrm{E}$ \\
\hline DF Tau & $1.5^{4}$ & 43.2 & $\mathrm{E} / \mathrm{FA}$ & $\mathrm{E}$ & $\mathrm{E}$ \\
\hline DG CrA & $0.5^{3}$ & 7.0 & $\mathrm{E} / \mathrm{FA}$ & & \\
\hline DG Tau & $2.4^{4,5}$ & $25.8 /-74.3$ & $\mathrm{E} / \mathrm{A}$ & $\mathrm{E} / \mathrm{A}$ & $\mathrm{E}$ \\
\hline DoAr 21 & $0.88^{6}$ & -0.26 & A & A & \\
\hline DoAr 24E N & $0.15^{3,6}$ & -0.04 & A & A & \\
\hline DoAr 24E S & $0.07^{3,6}$ & $1.7 /-0.1$ & $\mathrm{E} / \mathrm{FA}$ & $\mathrm{E} / \mathrm{FA}$ & $\mathrm{E}$ \\
\hline DoAr 44 & $0.54^{6}$ & 12.2 & $\mathrm{E}$ & & \\
\hline DR Tau & $1.3^{7}$ & 43.8 & $\mathrm{E}$ & $\mathrm{E}$ & $\mathrm{E}$ \\
\hline Elias 23 & $1.4^{3}$ & $15.1 /-22.3$ & $\mathrm{E} / \mathrm{A}$ & $\mathrm{E} / \mathrm{A}$ & $\mathrm{E}$ \\
\hline EC 82 & $0.6^{3}$ & $17^{*}$ & $\mathrm{E} / \mathrm{A}$ & $\mathrm{E} / \mathrm{A}$ & $\mathrm{E}$ \\
\hline EX Lup & $1.3^{8}$ & 20.8 & E & $\mathrm{E}$ & $\mathrm{E}$ \\
\hline FN Tau & $0.26^{9}$ & 1.3 & $\mathrm{E}$ & & \\
\hline FZ Tau & $1.0^{1}$ & 51.1 & $\mathrm{E}$ & & $\mathrm{E}$ \\
\hline GQ Lup & $1.0^{3}$ & 26.6 & $\mathrm{E}$ & & $\mathrm{E}$ \\
\hline Haro 1-4 S & $0.6^{3}$ & 10.0 & $\mathrm{E} / \mathrm{FA}$ & & $\mathrm{E}$ \\
\hline HBC 680 & $0.5^{3}$ & -0.3 & A & $\mathrm{A}$ & \\
\hline HD 135344 B & $2.3^{10}$ & 6.4 & $\mathrm{E}$ & $\mathrm{E}$ & $\mathrm{E}$ \\
\hline HD 142527 & $6.8^{10}$ & 40.9 & $\mathrm{E}$ & $\mathrm{E}$ & $\mathrm{E}$ \\
\hline HD $144432 \mathrm{~S}$ & $1.9^{5}$ & 10.1 & $\mathrm{E}$ & $\mathrm{E}$ & \\
\hline HD $144965 \mathrm{~A}$ & $0.5^{3}$ & $<0.7^{\mathrm{a}}$ & FA & & \\
\hline HD 176386 & $0.4^{11}$ & $<0.3^{\mathrm{a}}$ & FA & & \\
\hline IM Lup & $0.6^{3}$ & $<0.9^{\mathrm{a}}$ & FA & & \\
\hline IQ Tau & $0.5^{1}$ & $3.3 /-2.9$ & $\mathrm{E} / \mathrm{A}$ & & $\mathrm{E}$ \\
\hline IRS 46 & $0.6^{3}$ & -6.1 & A & A & \\
\hline IRS 48 & $2.0^{10}$ & 4.2 & $\mathrm{E} / \mathrm{FA}$ & $\mathrm{E} / \mathrm{FA}$ & $\mathrm{E}$ \\
\hline IRS 51 & $0.92^{6}$ & 12.1 & $\mathrm{E} / \mathrm{FA}$ & FA & \\
\hline LkHa 330 & $0.9^{9}$ & 4.1 & $\mathrm{E} / \mathrm{FA}$ & $\mathrm{E}$ & $\mathrm{E}$ \\
\hline $\mathrm{R} \mathrm{CrA}$ & $69^{13}$ & $-83 / 159 /-375$ & $\mathrm{E} / \mathrm{A}$ & $\mathrm{E} / \mathrm{A}$ & \\
\hline RNO 90 & $1.3^{3}$ & 44.7 & $\mathrm{E} / \mathrm{FA}$ & $\mathrm{E} / \mathrm{FA}$ & $\mathrm{E}$ \\
\hline RR Tau & $1.1^{13}$ & 1.7 & $\mathrm{E} / \mathrm{FA}$ & & \\
\hline RU Lup & $1.1^{14}$ & 32.1 & E & $\mathrm{E}$ & $\mathrm{E}$ \\
\hline RW Aur & $0.9^{5}$ & - & $\mathrm{E}$ & & \\
\hline RY Lup & $1.2^{3}$ & $8.6 /-3.0$ & $\mathrm{E} / \mathrm{A}$ & $\mathrm{E} / \mathrm{A}$ & \\
\hline S CrA N & $1.1^{2}$ & 42.5 & $\mathrm{E} / \mathrm{FA}$ & E & $\mathrm{E}$ \\
\hline S CrA S & $3.3^{2}$ & 74.3 & $\mathrm{E} / \mathrm{FA}$ & $\mathrm{E}$ & $\mathrm{E}$ \\
\hline Serp 32 & $2.8^{6}$ & $53.2 /-70.4$ & $\mathrm{E} / \mathrm{A}$ & & \\
\hline Serp 64 & $0.3^{6}$ & $4.0 /-3.0$ & $\mathrm{E} / \mathrm{A}$ & A & $\mathrm{E}$ \\
\hline SO 411 & $0.4^{3}$ & - & & & \\
\hline SR 9 A & $0.5^{2,6}$ & 5.3 & E & & \\
\hline SR 21 & $1.2^{15}$ & 2.8 & $\mathrm{E} / \mathrm{FA}$ & $\mathrm{E} / \mathrm{FA}$ & $\mathrm{E}$ \\
\hline SR $24 \mathrm{~A}$ & $1.4^{3,6}$ & $42.3 /-28.9$ & $\mathrm{E} / \mathrm{A}$ & A & $\mathrm{E}$ \\
\hline SR $24 \mathrm{~B} / \mathrm{C}$ & $0.6^{3,6}$ & $8.3 /-6.9^{*}$ & $\mathrm{E} / \mathrm{A}$ & A & \\
\hline SX Cha A & $0.6^{3}$ & 8.9 & $\mathrm{E}$ & & \\
\hline SY Cha & $0.5^{3}$ & $<3.8^{\mathrm{a}}$ & & & \\
\hline $\mathrm{Sz} 68 \mathrm{~A} / \mathrm{B}$ & $1.3^{3,16}$ & $5.8 /-6.3$ & $\mathrm{E} / \mathrm{A}$ & A & \\
\hline Sz $68 \mathrm{C}$ & $0.1^{3,16}$ & $8.3 /-6.9^{*}$ & A & $\mathrm{A}$ & \\
\hline T Cha & $1.2^{17}$ & -* & $\mathrm{A}$ & A & \\
\hline $\mathrm{T}$ CrA & $3.7^{13}$ & $7.0 /-18.7$ & $\mathrm{E} / \mathrm{A}$ & A & \\
\hline T Tau N & $11^{4}$ & ${ }^{*}$ & $\mathrm{E}$ & $\mathrm{E}$ & $\mathrm{E}$ \\
\hline T Tau S & $15^{4}$ & $-^{*}$ & $\mathrm{E} / \mathrm{A}$ & $\mathrm{E} / \mathrm{A}$ & $\mathrm{E}$ \\
\hline TW Cha & $0.5^{3}$ & 15.3 & E & & \\
\hline TW Hya & $0.3^{5}$ & 2.7 & $\mathrm{E}$ & & $\mathrm{E}$ \\
\hline TY CrA & $0.81^{13}$ & -0.3 & A & A & \\
\hline UX Tau & $3.9^{3}$ & - $^{*}$ & & & \\
\hline VSSG 1 & $1.0^{6}$ & $30.5 /-1.2$ & $\mathrm{E} / \mathrm{FA}$ & $\mathrm{E} / \mathrm{FA}$ & $\mathrm{E}$ \\
\hline VV CrA N & $0.7^{3,11}$ & -0.8 & A & A & $\mathrm{E}$ \\
\hline VV CrA S & $2.2^{3,11}$ & 26 & $\mathrm{E} / \mathrm{FA}$ & $\mathrm{E} / \mathrm{FA}$ & $\mathrm{E}$ \\
\hline VV Ser & $3.0^{13}$ & 15.2 & $\mathrm{E} / \mathrm{FA}$ & FA & \\
\hline
\end{tabular}

Table 2

(Continued)

\begin{tabular}{lccccc}
\hline \hline Source & $\begin{array}{c}M \text { Flux } \\
(\mathrm{Jy})\end{array}$ & $\begin{array}{c}P(8) \text { Flux } \\
\left(\mathrm{Jy} \mathrm{km} \mathrm{s}^{-1}\right)\end{array}$ & ${ }^{12} \mathrm{CO}$ & ${ }^{13} \mathrm{CO}$ & ${ }^{12} \mathrm{CO} 2-1$ \\
\hline VW Cha A & $0.4^{18}$ & 21.1 & $\mathrm{E}$ & & $\mathrm{E}$ \\
VZ Cha & $0.8^{18}$ & 35.5 & $\mathrm{E}$ & $\mathrm{E}$ \\
Wa Oph 6 & $0.9^{19}$ & 13.1 & $\mathrm{E} / \mathrm{FA}$ & $\mathrm{E}$ \\
WL 22 & $0.6^{3}$ & -0.0 & $\mathrm{~A}$ & $\mathrm{~A}$ & \\
WX Cha & $0.6^{3}$ & - & $\mathrm{E}$ & & $\mathrm{E}$ \\
\hline
\end{tabular}

Notes. E: emission; A: absorption; FA: foreground absorption, only present for $J<8$. Positive fluxes are in emission while negative fluxes denote absorption. * Strongly affected by telluric features.

a $3 \sigma$ upper limit on integrated line flux assuming a line width of $100 \mathrm{~km} \mathrm{~s}^{-1}$. References. (1) Luhman et al. 2006; (2) McCabe et al. 2006; (3) Derived from the CRIRES spectra; (4) Kenyon \& Hartmann 1995; (5) Hartmann et al. 2005; (6) Evans et al. 2009; (7) Salyk et al. 2008; (8) Goto et al. 2011; (9) Luhman et al. 2010; (10) Malfait et al. 1998; (11) Peterson et al. 2011; (12) Brown et al. 2012; (13) Hillenbrand et al. 1992; (14) Kessler-Silacci et al. 2006; (15) Brown et al. 2007; (16) Merín et al. 2008; (17) Alcala et al. 1993; (18) Luhman et al. 2008; (19) Padgett et al. 2006.

\subsection{Emission Profiles}

Sources with clean emission lines can be classified based on line width (Figure 5), with four distinct categories: (1) narrow, (2) broad single peaked, (3) broad, and (4) double peaked. The narrow category sources have line widths at base of less that $35 \mathrm{~km} \mathrm{~s}^{-1}$. Disks in this category are known to be face-on and/or transitional in nature (TW Hya, $\mathrm{LkH} \alpha$ 330, HD 135344 B, IRS 48), with CO likely arising from further out in the disk (Salyk et al. 2011). Lines which are slightly broader (FWHM 13-40 $\mathrm{km} \mathrm{s}^{-1}$ ) are generally part of the single-peaked line profile sample of Bast et al. (2011). These lines are characterized by a single central peak with broad wings and have large line-to-continuum values. The broad category objects retain a single peak but the contrast between the line width at the top and bottom (the $p$ value of Bast et al. 2011) decreases. The last group shows a double peaked structure.

Many of the $\mathrm{CO}$ emission lines in our sample are slightly asymmetric. To evaluate the magnitude of any asymmetries, the difference between the integrated flux in the blue and red sides of the lines was calculated for the stacked line profiles (see Figure 6). Where possible, the line center was determined from stellar radial velocity measurements. However, such measurements do not exist for many of the stars in the sample. In these cases, the line center was estimated from fitting and checked for large deviations from the known molecular cloud velocities. The lines show an overall tendency for excess emission on the blue side, regardless of line profile category. This trend affects lines of all widths indicating that it is unlikely to be a geometric effect. Telluric features introduce some uncertainty into the integrated fluxes but no systematic correlation with the telluric feature placement is seen. The most dramatic cases of this shift are those in the category which show strong emission only on the blue side of the line while absorption dominates the red side of the line. However, for the majority of the sample, the magnitude of the effect is generally small, $\sim 10 \%$ compared to the total flux.

\subsection{Absorption Profiles}

Absorption lines are common throughout the sample, with a detection rate of $67 \%$ (46 sources). Line widths range from the resolution limit of $\sim 3 \mathrm{~km} \mathrm{~s}^{-1}$ to tens of $\mathrm{km} \mathrm{s}^{-1}$. The energy 


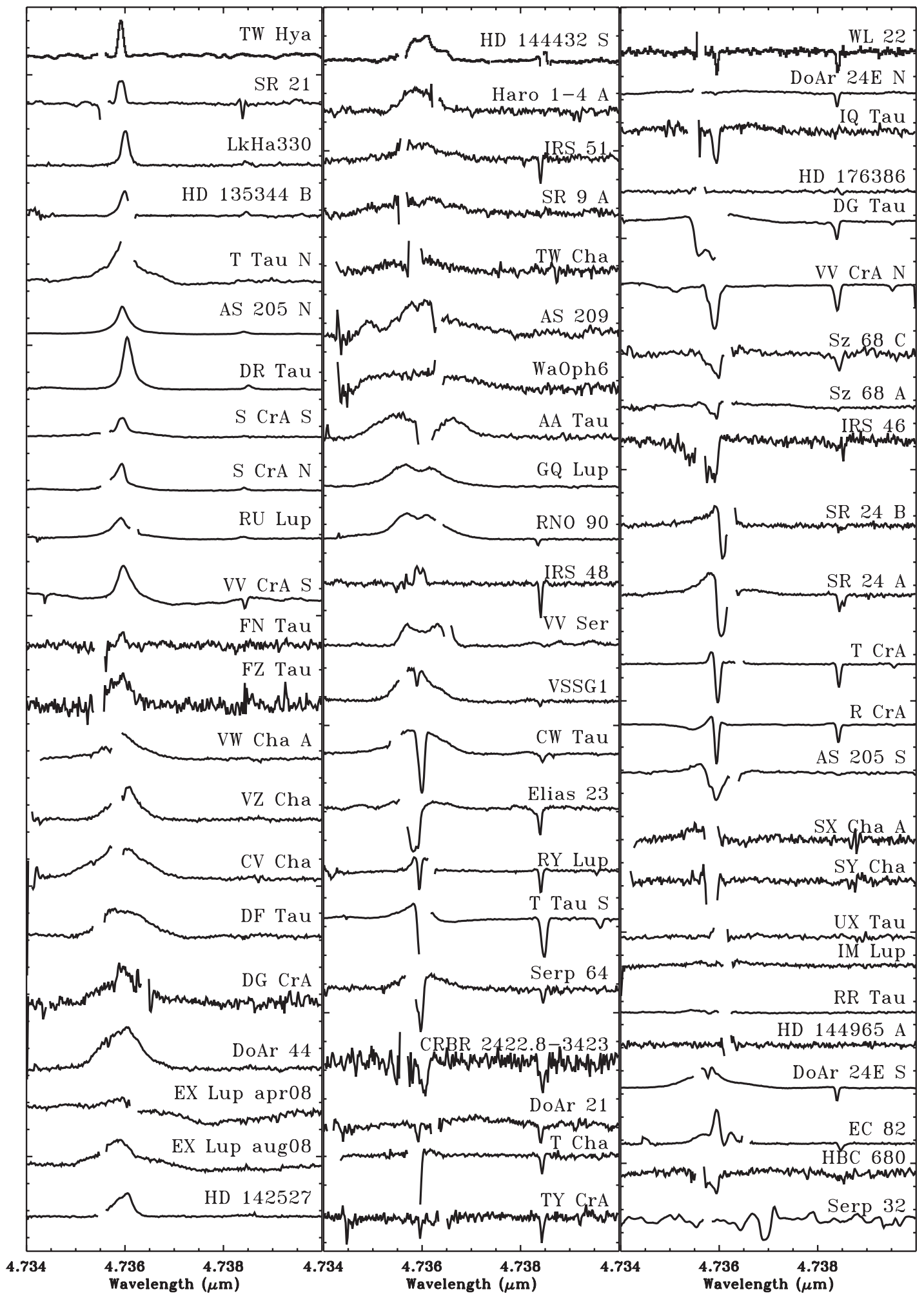

Figure 4. Gallery of the $P(8){ }^{12} \mathrm{CO}$ line at $4.7359 \mu \mathrm{m}$ with the ${ }^{13} \mathrm{CO} R(3)$ line seen in several spectra at $4.7383 \mu \mathrm{m}$ where available. Sources are ordered by similarity of line profile, roughly following the classification scheme in Table 3. RW Aur, SO 411, and WX Cha were not covered at these wavelengths and are missing from the plot.

levels with detectable absorption also vary from only the lowest $J$ levels to throughout the observed range, reflecting underlying temperature differences. In general, hotter gas produces broader lines, at a level much stronger than expected from thermal broadening (Figure 7). The lines occur both with and without emission. We divide the lines based on profile shape and temperature into four categories: (5) unresolved absorption,
(6) broad central absorption, (7) blue absorption, and (8) absorption with blue emission. The narrow absorption lines in category 5 are the most commonly seen absorption lines in our disk sample at $59 \%$ of all sources that show absorption $(27 / 46)$. For 21 of these sources, listed as FA (for foreground absorption) in Table 2, the lines are strongest in the lowest $J$ levels and disappear for higher energy transitions $(J>8)$. The remaining 


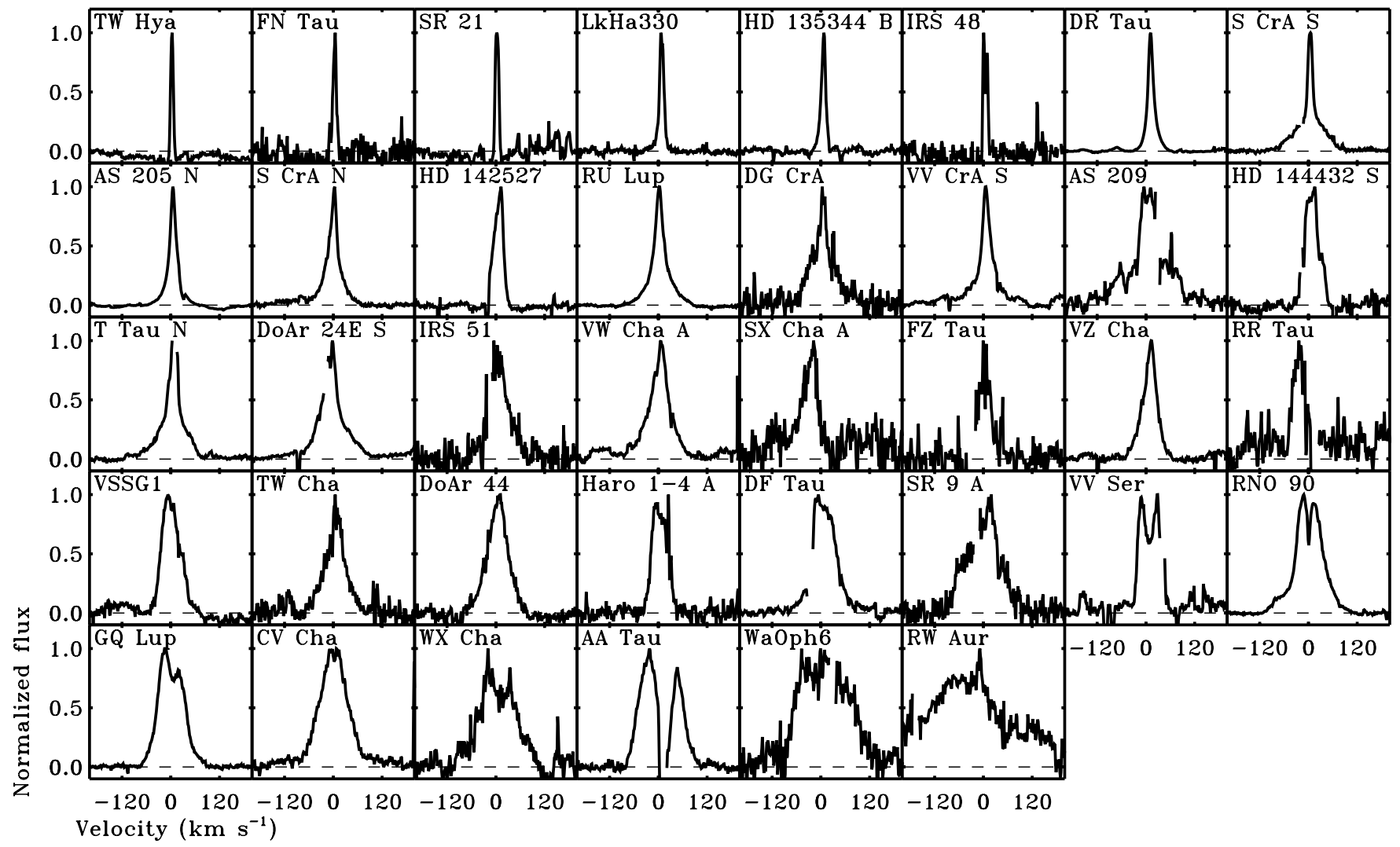

Figure 5. Normalized ${ }^{12} \mathrm{CO}$ line profiles of the sources with any emission lines free of absorption, arranged by increasing FWHM.

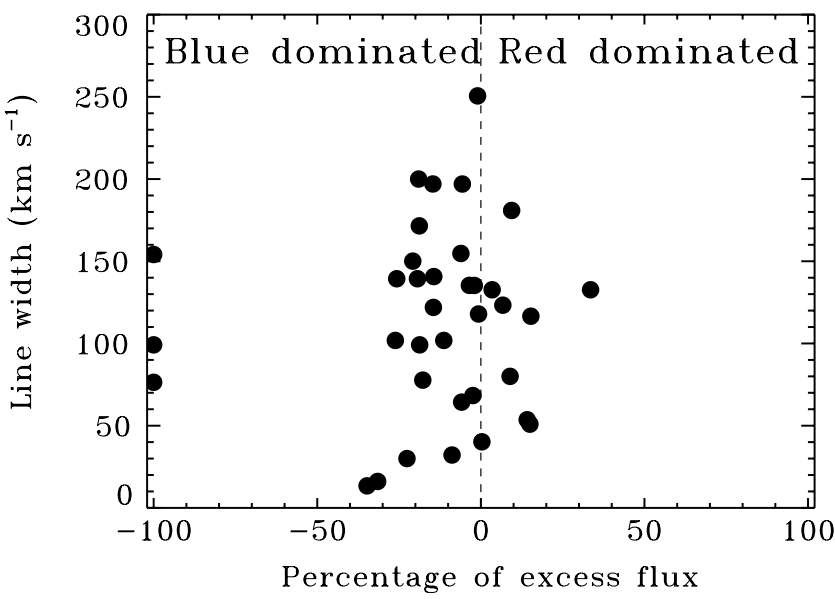

Figure 6. Percentage of excess blue/red flux compared to the total flux contrasted with the line width. The sources overall tend to have excess emission on the blue side of the line. This trend affects lines of all widths indicating that it is unlikely to be a geometric effect.

six sources in this category have hotter $\mathrm{CO}$ absorption and are listed in Table 3. Warm central absorption (category 6) is less common and almost always occurs with resolved line profiles. The $\gtrsim 100 \mathrm{~K}$ absorption is generally symmetric and centered at the stellar velocity (Figure 8). These warm absorption lines usually have FWHM of $\sim 10 \mathrm{~km} \mathrm{~s}^{-1}$ and occur together with broader emission lines. Absorption feature velocities generally agree well with stellar radial velocities (see Table 4). However, CW Tau and SR $24 \mathrm{~A}$ and B/C show a shift in the absorption line centers redward for higher energy lines.

Six sources belong to category 7 with clearly resolved blue wings in the absorption lines (Figure 9). These absorption

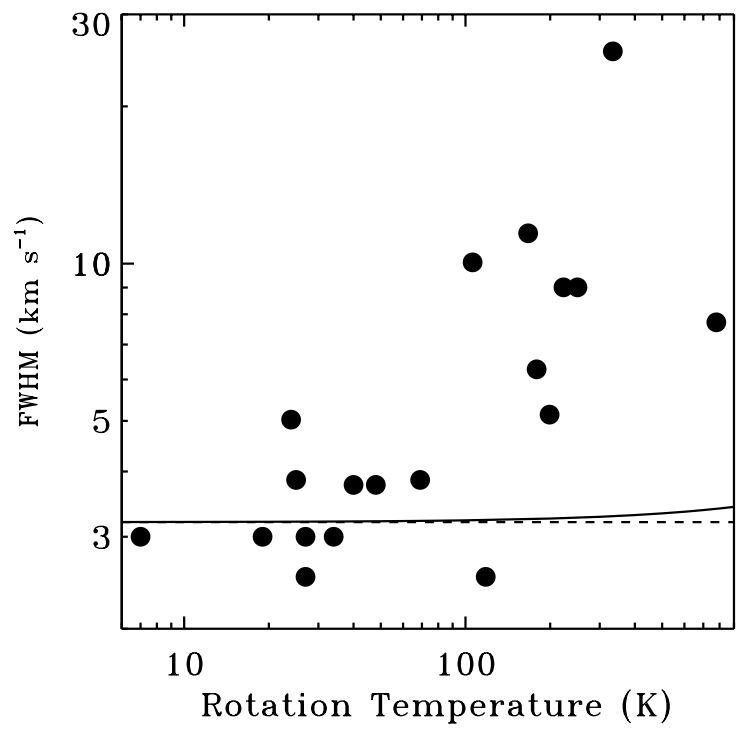

Figure 7. Comparison of FWHM with rotation temperature for absorption components. The resolution limit is marked by the dashed line. Unresolved lines generally arise from much colder gas than broader absorption lines. The solid line marks the width expected from thermal broadening convolved with the instrumental resolution.

features are usually seen throughout the covered $J$ range. The maximum velocities are less than $50 \mathrm{~km} \mathrm{~s}^{-1}$, except for IRS 46 where the gas reaches $\sim 60 \mathrm{~km} \mathrm{~s}^{-1}$. The final category (category 8) have lines that are characterized by emission on the blue side and absorption on the red (Figure 8).

\subsection{Absence of $\mathrm{CO}$ Lines}

Only six of our sources show no indication of CO lines in either emission or absorption. If CO is present, the lines are 
Table 3

Categorization of Line Profiles of Disk Sources

\begin{tabular}{|c|c|c|c|c|}
\hline (1) Narrow & (2) Broad Single Peaked & (3) Broad & (4) Double & No Lines \\
\hline $\begin{array}{l}\text { FN Tau } \\
\text { HD } 135344 \text { B } \\
\text { LkHa } 330 \\
\text { SR } 21 \\
\text { TW Hya }\end{array}$ & $\begin{array}{l}\text { AS } 205 \text { N } \\
\text { DR Tau } \\
\text { S CrA N } \\
\text { S CrA S } \\
\text { RU Lup } \\
\text { VV CrA S } \\
\text { VW Cha A } \\
\text { VZ Cha }\end{array}$ & $\begin{array}{l}\text { AS 209 } \\
\text { CV Cha } \\
\text { DF Tau } \\
\text { DG CrA } \\
\text { DoAr 24 E S } \\
\text { DoAr 44 } \\
\text { EX Lup } \\
\text { FZ Tau } \\
\text { HD 142527 } \\
\text { HD 144432 S } \\
\text { Haro 1-4 A } \\
\text { IRS 51 } \\
\text { RR Tau } \\
\text { RW Aur } \\
\text { SR 9 A } \\
\text { SX Cha A } \\
\text { TW Cha } \\
\text { VSSG 1 }\end{array}$ & $\begin{array}{l}\text { AA Tau } \\
\text { GQ Lup } \\
\text { IRS } 48 \\
\text { RNO90 } \\
\text { VV Ser } \\
\text { WaOph } 6 \\
\text { WX Cha }\end{array}$ & $\begin{array}{l}\text { HD } 144965 \text { A } \\
\text { HD } 176386 \\
\text { IM Lup } \\
\text { SO } 411 \\
\text { SY Cha } \\
\text { UX Tau }\end{array}$ \\
\hline $\begin{array}{l}\text { (5) Narrow Absorption } \\
\text { Only }\end{array}$ & $\begin{array}{l}\text { (6) Emission with Broad } \\
\text { Central Absorption }\end{array}$ & $\begin{array}{l}\text { (7) Blue } \\
\text { Absorption }\end{array}$ & $\begin{array}{l}\text { (8) Blue Emission/ } \\
\text { Red Absorption }\end{array}$ & Unclassifiable \\
\hline $\begin{array}{l}\text { CRBR 2422.8-3423 } \\
\text { DoAr } 21 \\
\text { DoAr } 24 \text { E N } \\
\text { T Cha } \\
\text { TY Cra } \\
\text { WL 22 }\end{array}$ & $\begin{array}{l}\text { CW Tau } \\
\text { Elias } 23^{\mathrm{b}} \\
\text { IQ Tau } \\
\text { RY Lup } \\
\text { Serp 64 } \\
\text { T Tau A }\end{array}$ & $\begin{array}{l}\text { DG Tau } \\
\text { HBC } 680 \\
\text { IRS } 46 \\
\text { Sz } 68 \text { A/B } \\
\text { Sz } 68 \text { C } \\
\text { VV CrA N }\end{array}$ & $\begin{array}{l}\text { AS } 205 \mathrm{~S} \\
\text { EC } 82 \\
\text { R CrA } \\
\text { SR } 24 \text { A } \\
\text { SR } 24 \text { B/C } \\
\text { T CrA }\end{array}$ & $\begin{array}{l}\text { Serp } 32 \\
\text { T Tau N }\end{array}$ \\
\hline
\end{tabular}

Notes.

${ }^{a}$ EX Lup was undergoing outburst during this time which affected the line profiles. A detailed examination of the $\mathrm{CO}$ emission based on this data can be found in Goto et al. (2011).

b The CO $v=2-1$ line profiles are double peaked so the underlying $v=1-0$ emission line profile could fall under category 4.

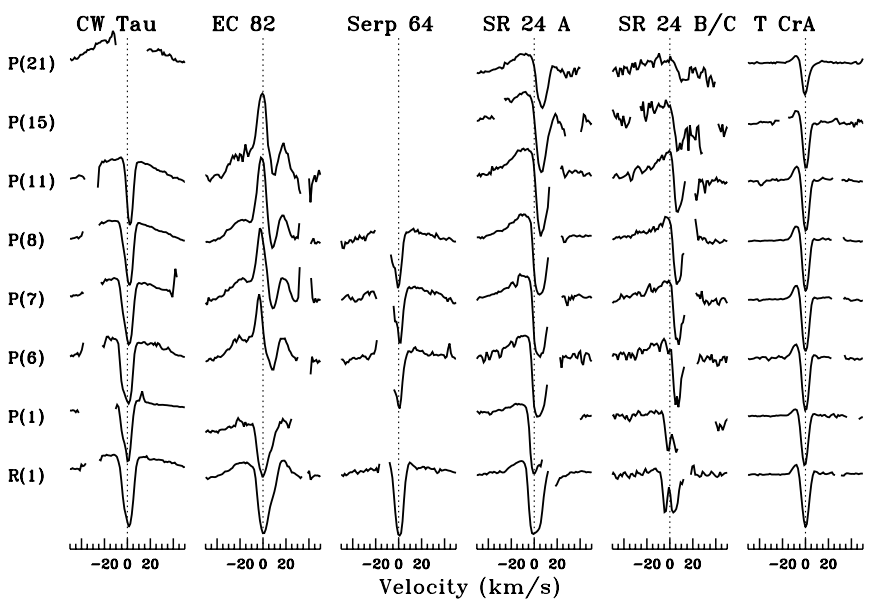

Figure 8. Sources with broad absorption lines. Most also have emission lines as well, generally stronger on the blue side of the line (e.g., SR 24, T CrA). The absorption line centers shift redward at higher $J$ for CW Tau, SR 24 A, and SR 24 B/C.

either weak or narrow enough to be lost in the telluric features $\left(<20 \mathrm{~km} \mathrm{~s}^{-1}\right)$. In all cases, the continuum is detected (generally with signal-to-noise ratio $(\mathrm{S} / \mathrm{N})$ of $\sim 50)$, indicating the presence of circumstellar dust in late-type stars. The $\mathrm{CO}$ non-detection from UX Tau A is particularly surprising as Salyk et al. (2009) show a clear detection within this band with NIRSPEC data. However, the telluric feature is poorly placed, covering -5 to $20 \mathrm{~km} \mathrm{~s}^{-1}$, which includes much of the line center. Also, the

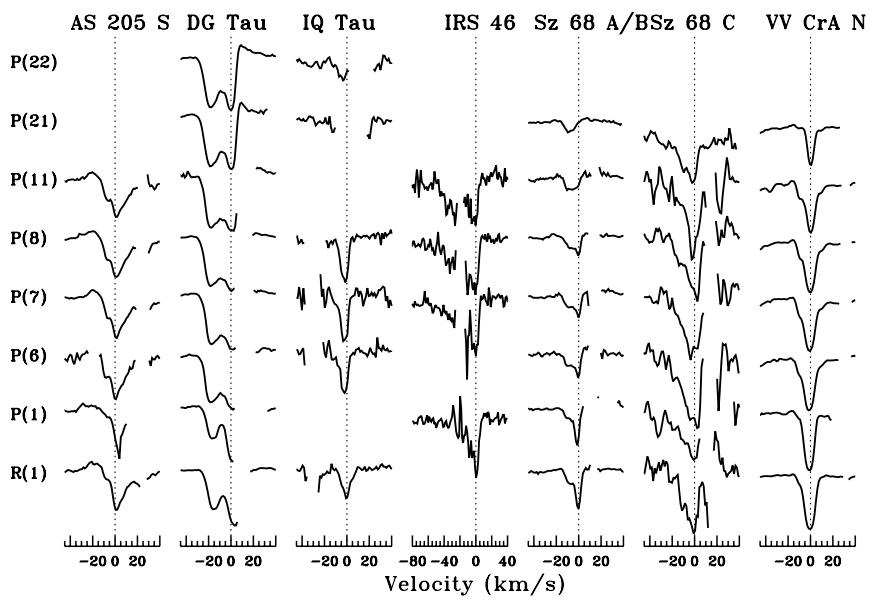

Figure 9. Sources with blue absorption wings up to high $J$, attributed to outflows. Most show maximum outflow velocities of $<30 \mathrm{~km} \mathrm{~s}^{-1}$ although IRS 46 reaches velocities of $\sim 60 \mathrm{~km} \mathrm{~s}^{-1}$. The VV CrA N data are from 2007 April; by 2007 August the blue absorption shoulder is no longer visible.

lack of CO from the IM Lup disk, which has been detected and imaged in $\mathrm{CO}$ millimeter emission out to several hundred AU is puzzling (van Kempen et al. 2007; Panić et al. 2009). HD 176386 is a B9 star (Torres et al. 2006) while HD 144965 A has a spectral type of $\mathrm{B} 3$ so both disks may simply have no $\mathrm{CO}$ close to the star due to high photodissociation rates. In general, very few sources have no $\mathrm{CO}$ visible in the spectrum. 
Table 4

Radial Velocities

\begin{tabular}{|c|c|c|c|c|}
\hline Source & $\begin{array}{l}V_{*}(\text { Helio }) \\
\left(\mathrm{km} \mathrm{s}^{-1}\right)\end{array}$ & $\begin{array}{l}V_{*}(\mathrm{LSR}) \\
\left(\mathrm{km} \mathrm{s}^{-1}\right)\end{array}$ & $\begin{array}{c}V_{\mathrm{CO}}(\mathrm{LSR}) \\
\left(\mathrm{km} \mathrm{s}^{-1}\right)\end{array}$ & Ref. \\
\hline AA Tau & $16.1 \pm 2$ & 5.6 & 9.0 & 1 \\
\hline AS $205 \mathrm{~N}$ & $-9.4 \pm 1.5$ & 2.0 & 5.0 & 2 \\
\hline AS 209 & $-8.5 \pm 1$ & 5.0 & 5.7 & 2 \\
\hline CV Cha & $16.1 \pm 1.3$ & 5.5 & 3.0 & 3 \\
\hline CW Tau & $14.5 \pm 2$ & 5.6 & -8.7 & 1 \\
\hline DF Tau & $12 \pm 5$ & 2.1 & 8.7 & 1 \\
\hline DG CrA & $-1.8 \pm 0.4$ & 5.4 & 0.7 & 3 \\
\hline DG Tau & $19.3 \pm 2.7$ & 9.5 & -0.4 & 4 \\
\hline DoAr 24 E N & $-8.0 \pm 2.5$ & 2.3 & 3.5 & 2 \\
\hline DoAr 44 & $-5.9 \pm 0.4$ & 4.7 & 11.4 & 2 \\
\hline EX Lup & $-1.5 \pm 2.3$ & 3.6 & 3.3 & 2 \\
\hline FN Tau & $14.9 \pm 0.4$ & 6.1 & 4.6 & 5 \\
\hline GQ Lup & $-3.2 \pm 0.6$ & 2.8 & 6.0 & 2 \\
\hline Haro 1-4 A & $-7.6 \pm 0.1$ & 2.8 & 5.4 & 2 \\
\hline HD $135344 \mathrm{~B}$ & $-3 \pm 3$ & 1.3 & 7.4 & 6 \\
\hline RU Lup & $-0.9 \pm 1.1$ & 4.6 & 2.0 & 2 \\
\hline RW Aur & $16.0 \pm 1.7$ & 6.5 & -0 & 4 \\
\hline RY Lup & $-2.0 \pm 0.8$ & 2.9 & -0.0 & 2 \\
\hline $\mathrm{S} \mathrm{CrA}$ & $-0.3 \pm 1.7$ & 7.1 & 3.3 & 2 \\
\hline Sz 68 & $-2.3 \pm 0.6$ & 3.9 & 4.5 & 2 \\
\hline T Cha & $14.0 \pm 1.3$ & 4.1 & 6.0 & 3 \\
\hline T Tau N & $19.1 \pm 2$ & 7.6 & 8.7 & 1 \\
\hline TW Cha & $17.8 \pm 1$ & 7.0 & 5.0 & 3 \\
\hline TW Hya & $12.4 \pm 0.2$ & 3.0 & 3.3 & 7 \\
\hline VW Cha A & $17.2 \pm 0.5$ & 6.6 & 6.0 & 3 \\
\hline VZ Cha & $18.0 \pm 1.1$ & 7.3 & 12.7 & 2 \\
\hline
\end{tabular}

References. (1) Herbig \& Bell 1988; (2) Melo 2003; (3) Guenther et al. 2007; (4) White \& Hillenbrand 2004; (5) Muzerolle et al. 2003; (6) Dunkin et al. 1997; (7) Setiawan et al. 2008.

\section{CO EXCITATION}

\subsection{Rotational Temperatures}

The high resolving power of CRIRES allows for the detection of low-contrast emission lines, leading to an enhanced potential for measuring the rotational temperatures and accurate column densities of the weak optically thin isotopologues. In this section, we take advantage of this to compare the rotational temperatures of ${ }^{12} \mathrm{CO},{ }^{13} \mathrm{CO} v=1-0$, and ${ }^{12} \mathrm{CO} v=2-1$ transitions.

A linear fit to the continuum was determined for each line, using an uncontaminated continuum within $300 \mathrm{~km} \mathrm{~s}^{-1}$ of the line center and $50 \mathrm{~km} \mathrm{~s}^{-1}$ greater than the line width. For accurate measurements of integrated line fluxes, we constructed template line profiles by stacking isolated lines free of strong telluric absorption. Absorption components were removed using a Gaussian profile. The integrated line fluxes were determined by scaling the template to each line (or a superposition of templates in case of line blends) using Levenberg-Marquardt least-squares minimization. This method proved advantageous to direct integration of the line flux: while direct integration works well for strong lines, it is problematic for multi-component line shapes, weak lines, and lines affected by telluric absorption. Each line fit was examined by eye and those that clearly did not recover a meaningful line flux due to strong telluric residuals or line blending were removed from further analysis.

The fluxes are put into rotational diagrams to determine rotational temperatures and column densities. Based on an assumed Boltzmann distribution, the observed flux $F_{J}^{\prime}$ is such
Table 5

Summary of ${ }^{13} \mathrm{CO}$ Rotation Diagrams

\begin{tabular}{lcccc}
\hline \hline Source & $\begin{array}{c}T\left({ }^{13} \mathrm{CO}\right) \\
(\mathrm{K})\end{array}$ & $\begin{array}{c}\text { Opt. Thin } \\
\text { Radius }(\mathrm{AU})^{\mathrm{a}}\end{array}$ & $\begin{array}{c}N_{\mathrm{CO}, 100 \mathrm{AU}^{2}} \\
\left(10^{17} \mathrm{~cm}^{-2}\right)\end{array}$ & $\begin{array}{c}\text { Gas Mass } \\
\left(10^{-4} M_{\odot}\right)\end{array}$ \\
\hline AS 205 N & 480 & 0.8 & 1.2 & 0.0058 \\
DoAr 24E S & 490 & 0.35 & 0.089 & 0.00045 \\
DR Tau & 570 & 1.3 & 0.95 & 0.0048 \\
HD 135344 B & 250 & 5.0 & 41.0 & 0.21 \\
HD 142527 & 420 & 3.1 & 0.26 & 0.088 \\
IRS 48 & 270 & 5.0 & 40.0 & 0.20 \\
LkH $\alpha$ 330 & 240 & 13 & 460.0 & 2.3 \\
RU Lup & 310 & 4.4 & 25.0 & 0.12 \\
S CrA N & 380 & 5.6 & 17.0 & 0.086 \\
S CrA S & 280 & 8.9 & 120.0 & 0.60 \\
SR 21 & 220 & 18 & 770.0 & 3.9 \\
T Tau N & 640 & 0.8 & 0.54 & 0.0027 \\
\hline
\end{tabular}

Note. ${ }^{\text {a }}$ Where $A_{\mathrm{emit}}=\pi R_{\mathrm{emit}}^{2}$.

that

$$
\frac{4 \pi F_{J}^{\prime}}{h c v_{J} \Omega g_{J} A_{J i}}=\frac{N_{\text {tot }}}{Q\left(T_{\text {rot }}\right)} e^{-E_{J} / k T_{\text {rot }}},
$$

where $\Omega$ is the emitting area, $A_{J i}$ is the Einstein $A$ coefficient of each level $i$ to which the $J$ level can decay, $g_{J}$ is the statistical weight of each level (i.e., $2 J+1), N_{\text {tot }}$ is the column density, $Q\left(T_{\text {rot }}\right)$ is the partition function, $E_{J}$ is the energy of the transition, and $T_{\text {rot }}$ is the rotation temperature. For optically thin, isothermal gas in thermodynamic equilibrium (TE), rotation diagrams produce a straight line with a slope of inverse temperature and an intercept proportional to the total mass of gas, but are insensitive to emitting area. In the optically thick limit, temperature and emitting area are degenerate, while the rotation diagrams are insensitive to the total column density. Thus, curved rotation diagrams can be a sign of high line optical depths varying with $J$, although multiple temperature components can also cause curvature. When both optically thick and thin tracers are used, it may be possible to constrain all three parameters, $N, T_{\text {rot }}$, and $\Omega$, if both isotopologues arise from the same region, but, as shown below, this is not the case.

$$
\text { 4.1.1. }{ }^{13} \mathrm{CO}
$$

${ }^{13} \mathrm{CO}$ is detected in emission from 27 sources and suitable line fits (minimum of good fits to five clean lines) could be determined for 12 sources (see Table 5). The sources consist almost exclusively of a mixture of transitional disks and the broad single peaked sources of Bast et al. (2011). The lines are relatively weak compared to the ${ }^{12} \mathrm{CO} v=1-0$ and $v=2-1$ lines with fluxes down by factors of $\sim 10$ and $\sim 4$, respectively.

The ${ }^{13} \mathrm{CO}$ fluxes are distributed along a straight line within the errors in the rotational diagrams - consistent with single temperature optically thin emission (see Figure 11 for examples). Possible exceptions include AS $205 \mathrm{~N}$ and DR Tau where some curvature may be apparent in the highest and lowest $J$ levels. For the case of DR Tau, determination of the ${ }^{13} \mathrm{CO} / \mathrm{C}^{18} \mathrm{O}$ flux ratios indicates optical depths of the ${ }^{13} \mathrm{CO}$ lines of only $0.3 \pm$ 0.2 (Bast et al. 2011). The emitting radius at which the ${ }^{13} \mathrm{CO}$ is optically thin is listed in Table 5, assuming a circular slab model with uniform temperature and mass.

Optically thin linear fits uniformly reveal temperatures between 200 and $600 \mathrm{~K}$ with the majority in the 200-400 K range (Figure 10), consistent with the analysis of Bast et al. (2011) for a subset of the sources. This is significantly lower than has been previously reported for $\mathrm{CO}$ fundamental band 

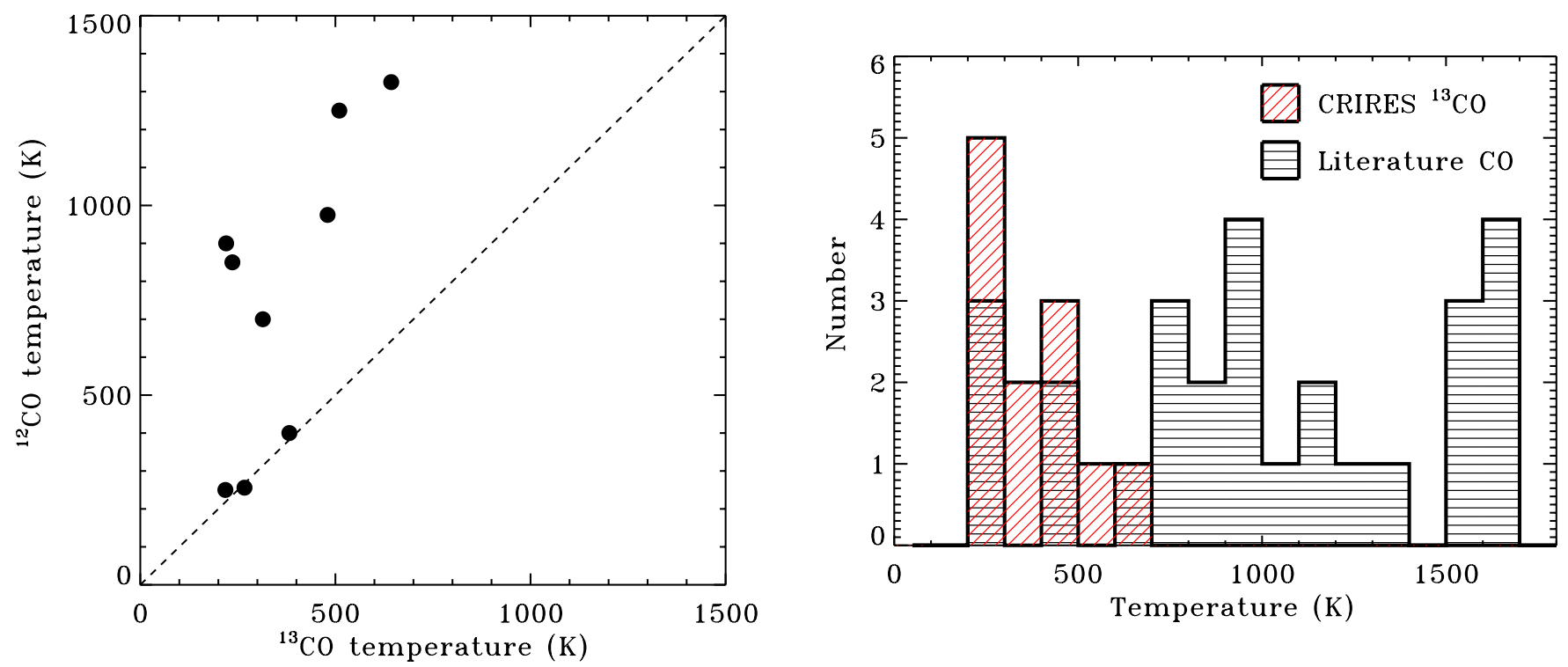

Figure 10. Left: ${ }^{13} \mathrm{CO}$ temperatures derived from optically thin fits of disk emission sources compared to temperatures derived from fitting the ${ }^{12} \mathrm{CO}$ lines. Right: ${ }^{13} \mathrm{CO}$ temperatures derived from optically thin fits compared to CO temperatures reported in the literature (Salyk et al. 2009, 2011). The three literature sources with ${ }^{12} \mathrm{CO}$ temperatures in the $200-300 \mathrm{~K}$ range are all sources for which ${ }^{13} \mathrm{CO}$ was previously detected.

(A color version of this figure is available in the online journal.)
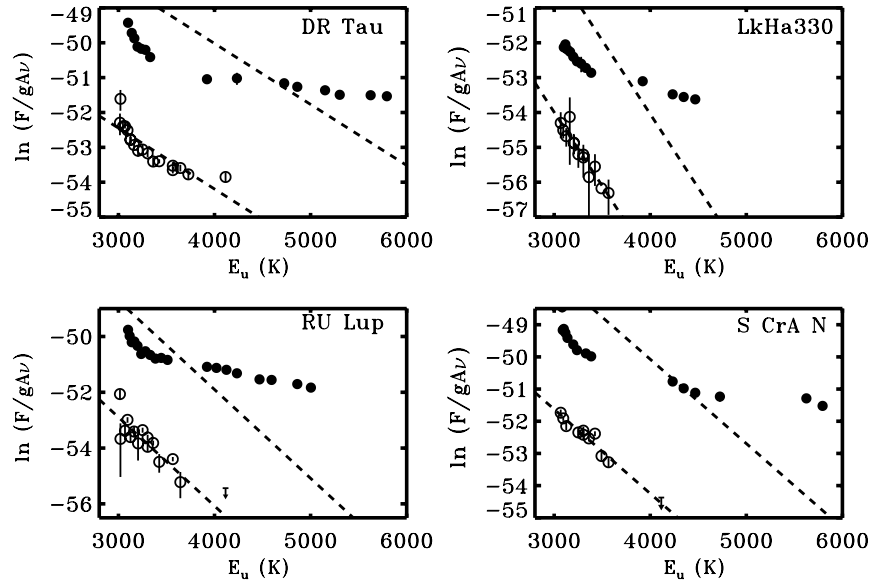

Figure 11. Rotational diagrams of ${ }^{12} \mathrm{CO}$ (filled circles) and ${ }^{13} \mathrm{CO}$ (open circles). A linear fit was made to the ${ }^{13} \mathrm{CO}$, lower dashed line, and was scaled up by an isotope ratio of 65 to compare to the expected ${ }^{12} \mathrm{CO}$ if optically thin. The high $J$ lines are clearly significantly underpredicted and require a hotter gas component to fit. The lower $J$ lines are likely underpredicted due to optical depth effects.

temperatures, generally determined from optically thick ${ }^{12} \mathrm{CO}$, which may trace different gas. Literature values are included in Figure 10. In the optically thin case, the range of energies covered should have no effect on the linear fit. For the sources with detectable ${ }^{13} \mathrm{CO}$, lines are detected generally up to $P / R(15)$ $\left(E_{u}=3656 \mathrm{~K} / 3826 \mathrm{~K}\right)$ and up to $R(23)\left(E_{u}=4722 \mathrm{~K}\right)$ in AS $205 \mathrm{~N}$.

The fact that the ${ }^{13} \mathrm{CO}$ lines appear optically thin makes it possible to directly correlate the $y$-intercept and total ${ }^{13} \mathrm{CO}$ gas mass. Table 5 includes the inferred $\mathrm{CO}$ column densities assuming an emitting area of $100 \mathrm{AU}^{2}(\sim 5 \mathrm{AU}$ radius) and ${ }^{12} \mathrm{CO} /{ }^{13} \mathrm{CO}=65$. The exact area for which the ${ }^{13} \mathrm{CO}$ emission becomes optically thin depends on the specific sources and also on the assumed local line profile, taken here to have a total broadening of $2 \mathrm{~km} \mathrm{~s}^{-1}$, the sound speed of $\mathrm{H}_{2}$ at $1000 \mathrm{~K}$, in agreement with Herczeg et al. (2011) and Salyk et al. (2011).
Larger broadening parameters result in more optically thin gas while smaller broadening parameters have the opposite effect.

For a few sources, the inferred $\mathrm{CO}$ column densities are close to $10^{20} \mathrm{~cm}^{-2}$ (SR 21, $\mathrm{LkH} \alpha 330$ ) within the assumed 5 AU radius, which would imply values of $N_{\mathrm{H}}>10^{24} \mathrm{~cm}^{-2}$ for a standard conversion factor of $\mathrm{CO} / \mathrm{H}_{2}=10^{-4}$. These column densities are very large compared to the surface column densities of $N_{\mathrm{H}} \sim 10^{21}-10^{23} \mathrm{~cm}^{-2}$ that are expected down to the layer where the $5 \mu \mathrm{m}$ continuum becomes optically thick (e.g., Aikawa et al. 2002; Gorti \& Hollenbach 2008; Woitke et al. 2009). Another way to look at this problem is to derive total gas masses from the ${ }^{13} \mathrm{CO}$ data. Values computed under the optically thin assumption (but independent from any assumed emitting area) are included in Table 5. For some sources, the masses are $>10^{-4} M_{\odot}$, implying that a significant fraction of the disk mass would be contained in just the surface layers of the inner few AU of the disk, under these assumptions. One possible solution to this conundrum of the large inferred column densities and masses is that the dust grains are settled to the midplane so that the gas/dust ratio is much larger than the standard value, which allows us to look deeper into the disk at $5 \mu \mathrm{m}$. A correlation between mid-IR spectral energy distribution (SED) slopes, a tracer of settling, and CO equivalent widths indicates that dust settling may increase the observable $\mathrm{CO}$ (see Section 5.4). Another possibility is that UV or IR radiative excitation and resonant scattering contributes to the ${ }^{13} \mathrm{CO}$ lines fluxes (see Section 4.1.2), in which case the inferred column densities and masses are upper limits.

The results from optically thin fits of the ${ }^{13} \mathrm{CO}$ gas, detected here with a much higher frequency than in previous studies, imply a different location for at least some of the $\mathrm{CO}$ than indicated from studies of ${ }^{12} \mathrm{CO}$. The low temperatures and large emitting areas suggest that much of the ${ }^{13} \mathrm{CO}$ arises from either larger disk radii or deeper into the disk.

$$
\text { 4.1.2. }{ }^{12} \mathrm{CO}
$$

The ${ }^{12} \mathrm{CO}$ lines throughout our sample show curvature in the rotation diagram which has been attributed to optical depth 


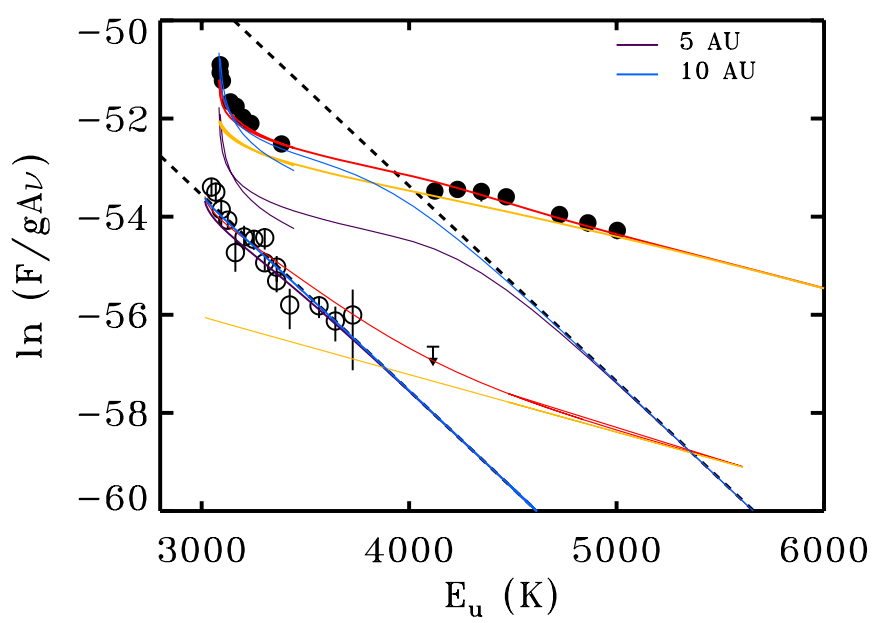

Figure 12. Rotational diagram for ${ }^{12} \mathrm{CO}$ (filled circles) and ${ }^{13} \mathrm{CO}$ (open circles) from HD $135344 \mathrm{~B}$. The blue and purple lines are isothermal slab models based on the rotational temperature and mass determined from the optically thin fits to the ${ }^{13} \mathrm{CO}\left(250 \mathrm{~K}, 8 \times 10^{16} \mathrm{~cm}^{-2}\right)$ for two emitting radii. The yellow line is a higher temperature model $\left(850 \mathrm{~K}, N\left({ }^{12} \mathrm{CO}\right)=10^{17.5} \mathrm{~cm}^{-2}\right.$ column density) to fit the high $J^{12} \mathrm{CO}$ and the red line is this model combined with the $5 \mathrm{AU}$ radius ${ }^{13} \mathrm{CO}$ model.

(A color version of this figure is available in the online journal.)

effects (Blake \& Boogert 2004). They look qualitatively similar to previously published rotation diagrams from disks (Salyk et al. 2009; Najita et al. 2003). In cases where the sources had been previously observed at lower spectral resolution (e.g., NIRSPEC; Salyk et al. 2009), benchmark tests ensured that our CRIRES rotation diagrams quantitatively agree with previous observations.

In theory, optically thin ${ }^{13} \mathrm{CO}$ can constrain the degeneracies in modeling the optically thick ${ }^{12} \mathrm{CO}$ emission, assuming that the emission arises from the same gas. However, the low ${ }^{13} \mathrm{CO}$ temperatures are incapable of explaining the observed ${ }^{12} \mathrm{CO}$ high $J$ lines in most cases, indicating the presence of a warm component seen in the ${ }^{12} \mathrm{CO}$ (Figure 11). We examine HD $135344 \mathrm{~B}$ in detail to determine whether a two temperature model is capable of fitting this behavior (Figure 12). We use a simple slab model which calculates the optical depth in each transition (see, e.g., Salyk et al. 2009; Brown 2008). The ${ }^{13} \mathrm{CO}$ data are best fitted with $T_{\text {rot }}=250 \mathrm{~K}, N\left({ }^{13} \mathrm{CO}\right)=8 \times 10^{16} \mathrm{~cm}^{-2}$ and an emitting region with a radius of $5 \mathrm{AU}$ to ensure that the ${ }^{13} \mathrm{CO}$ emission is optically thin. Using such a large radius and multiplying the column density by 65 , the low ${ }^{13} \mathrm{CO}$ temperature is incapable of explaining the high $J P(20-27){ }^{12} \mathrm{CO}$ lines which are clearly detected (formal errors are smaller than the points; Figure 11). A smaller, hotter $(850 \mathrm{~K})$ region of ${ }^{12} \mathrm{CO}$ emission needs to be added to fit the ${ }^{12} \mathrm{CO}$ data. We conclude that a two temperature model (black line) is capable of fitting the data as the expected ${ }^{13} \mathrm{CO}$ fluxes from the warm ${ }^{12} \mathrm{CO}$ component are below our detection thresholds if close to optically thin.

Previous studies have also had difficulty reconciling the ${ }^{12} \mathrm{CO}$ and ${ }^{13} \mathrm{CO}$ line fluxes for some sources. Salyk et al. (2009) found a similarly low temperature of $250-350 \mathrm{~K}$ for ${ }^{13} \mathrm{CO}$ in SR 21 . This is compatible with the ${ }^{12} \mathrm{CO}$ lines due to a lack of detected high $J$ lines for that source. However, the required mass of $6 \times 10^{25} \mathrm{~g}$ of $\mathrm{CO}$ (gas mass of $3 \times 10^{-4} M_{\odot}$ ) and emitting area of $78 \mathrm{AU}^{2}$ are large, although a gas location at radii as large as $7 \mathrm{AU}$ is confirmed by spectro-astrometry (Pontoppidan et al. 2011a). Blake \& Boogert (2004) were unable to explain the high relative fluxes from ${ }^{13} \mathrm{CO}$ in $\mathrm{AB}$ Aurigae and Brittain et al. (2009) found anomalously high ${ }^{13} \mathrm{CO}$ line fluxes from the disk around A-type star HD 100546, with an apparent ratio of ${ }^{12} \mathrm{CO} /{ }^{13} \mathrm{CO}$ of 4 rather than the interstellar ratio of $\sim 65$. In both cases, non-thermal processes were invoked to explain the discrepancy. Blake \& Boogert (2004) find that resonant scattering of a large fraction of the IR continuum photons can reproduce the AB Aur fluxes. Brittain et al. (2009), on the other hand, suggest that UV pumping is the primary excitation mechanism and the overabundance of ${ }^{13} \mathrm{CO}$ is the result of additional sources of opacity in the disk.

IR resonant scattering (IR pumping) of ${ }^{13} \mathrm{CO}$ primarily enhances the low energy $J$ lines, reflecting the thermal distribution of the lower vibrational level population in the excited state. Optical depth effects may reduce the strength of this component in the ${ }^{12} \mathrm{CO}$ lines. Excitation via IR pumping is expected to be particularly effective for disk wind sources and transitional disks, explaining why we see ${ }^{13} \mathrm{CO}$ mostly from these sources. Winds lift material above the disk where the $\mathrm{CO}$ molecules are more exposed to radiation. In transitional disks, the lower $5 \mu \mathrm{m}$ continuum optical depth may result in less shielding from the dust and thus enhanced absorption. Resonant scattering is primarily dependent on the received flux and would imply column densities several orders of magnitude smaller to reproduce our observed line fluxes.

\subsection{Vibrational Excitation}

Vibrational lines from CO $v=2-1, v=3-2$, and $v=4-3$ are seen from a subset of sources. CO $v=2-1$ lines are the strongest and thus most commonly detected. These higher energy lines are likely pumped by UV flux as the upper level temperatures of $\sim 7000 \mathrm{~K}$ are too high for collisional excitation by the $500-1000 \mathrm{~K}$ gas seen in the ${ }^{12} \mathrm{CO}$ lines.

A vibrational flux ratio was calculated for each source based on the $v=2-1$ flux divided by the $v=1-0$ flux for the equivalent rotational line (Table 6). UV pumping does not induce large changes in population distribution in the different rotational states since the selection rule only allows quantum number changes of $\Delta J \pm 1$. Thus, the shape of the $v=2-1$ rotational diagram is similar to the $v=1-0$ lines and the offset between the two reflects the difference in upper state population. Measuring the vibrational flux ratios of equivalent $J$ rotation lines thus traces the population difference. Typically, five flux ratios were found and the median was taken for the final value to remove outliers. The vibrational flux ratios range from 0.05 to 0.5 and upper limits were calculated for high $\mathrm{S} / \mathrm{N}$ spectra where the CO $v=2-1$ lines were not detected.

The observed vibrational flux ratio generally increases with accretion luminosity (Figure 13). The accretion luminosity is determined based on simultaneous measurements of the $\operatorname{Pf} \beta$ line which is covered serendipitously in the CRIRES spectrum using the conversion to accretion luminosity as given in Salyk et al. (2013). IRS 48 is an outlier in the plot, probably due to its higher spectral type of A0 resulting in a photospheric UV flux rather than accretion luminosity pumping the $v=2-1$ lines and the $30 \mathrm{AU}$ gas location (Brown et al. 2012) reducing collisional excitation, and is therefore excluded from the linear fit.

We modeled the effects of a stellar UV field on the $\mathrm{CO}$ emission in a UV excitation model similar to Brittain et al. (2007). UV fluorescent pumping of the CO vibrational lines in the fundamental band involves excitation by UV photons from the $X^{1} \Sigma^{+}$ground electronic state to the $A^{1} \Pi$ excited electronic state. The decay from the excited electronic state to the ground state can increase the vibrational energy of the molecule by 


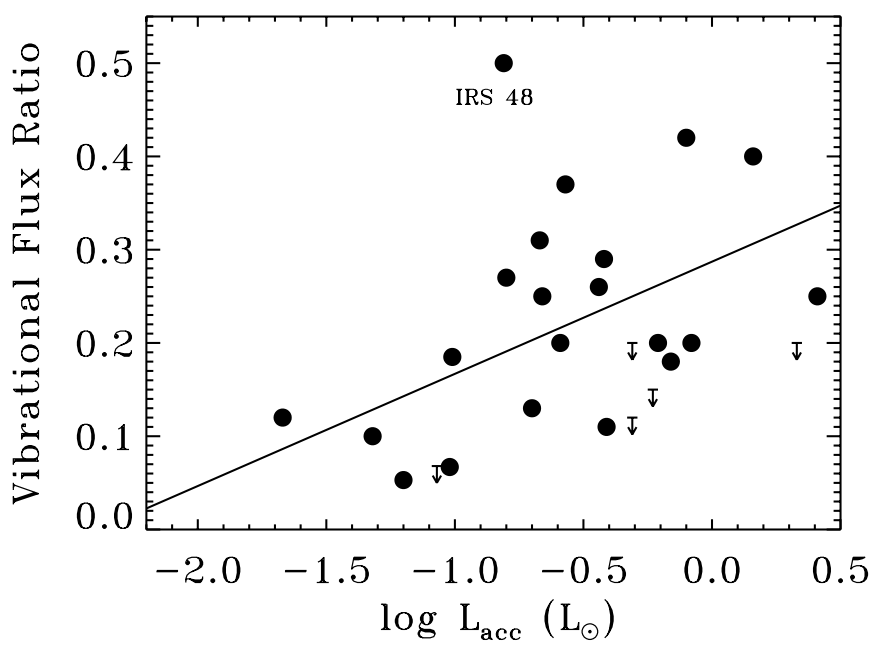

Figure 13. Flux ratio in vibrationally excited levels derived from the $2-1 / 1-0$ flux ratios vs. accretion luminosity determined from simultaneous $\operatorname{Pf} \beta$ emission. The accretion luminosity in the UV drives UV fluorescent pumping which is reflected in increased CO $v=2-1$ line fluxes. IRS 48 is an outlier in the plot likely due to its higher spectral type of A0 resulting in photospheric UV flux rather than accretion luminosity pumping the $v=2-1$ lines, and is therefore excluded from the linear fit.

Table 6

Vibrational Flux Ratios

\begin{tabular}{lc}
\hline \hline Source & $\begin{array}{c}\text { Vibrational Flux Ratio } \\
(\mathrm{CO} v=2-1 / v=1-0)\end{array}$ \\
\hline AA Tau & 0.1 \\
AS 205 N & 0.2 \\
AS 209 & $<0.2$ \\
CV Cha & 0.25 \\
CW Tau & 0.26 \\
DF Tau & 0.37 \\
DoAr 24E S & 0.12 \\
DoAr 44 & $<0.068$ \\
DR Tau & 0.20 \\
FZ Tau & $<0.15$ \\
GQ Lup & 0.19 \\
HD 135344 B & 0.067 \\
HD 144432 S & 0.12 \\
IRS 48 & 0.5 \\
LkH $\alpha 330$ & 0.11 \\
RNO 90 & 0.18 \\
RU Lup & 0.29 \\
S CrA N & 0.42 \\
S CrA S & 0.2 \\
TW Hya & 0.053 \\
WaOph 6 & 0.25 \\
VSSG 1 & 0.27 \\
VV CrA S & 0.4 \\
VV Ser & $<0.2$ \\
VW Cha A & 0.13 \\
VZ Cha & 0.31 \\
\hline
\end{tabular}

populating higher vibrational levels in the ground electronic state than would be expected from purely thermal excitation. We assume that the system is in a steady state and balance pumping into the $A^{1} \Pi$ state from the incident $\mathrm{UV}$ radiation field with spontaneous emission out of the $A^{1} \Pi$ state. Spontaneous emission within the $X^{1} \Sigma^{+}$ground state vibrational levels is also included. We expanded the vibrational states included in the model to $35 X^{1} \Sigma^{+}$ground levels and $25 A^{1} \Pi$ levels; rotational levels are not explicitly taken into account. For this purpose, we computed the oscillator strengths and Einstein- $A$

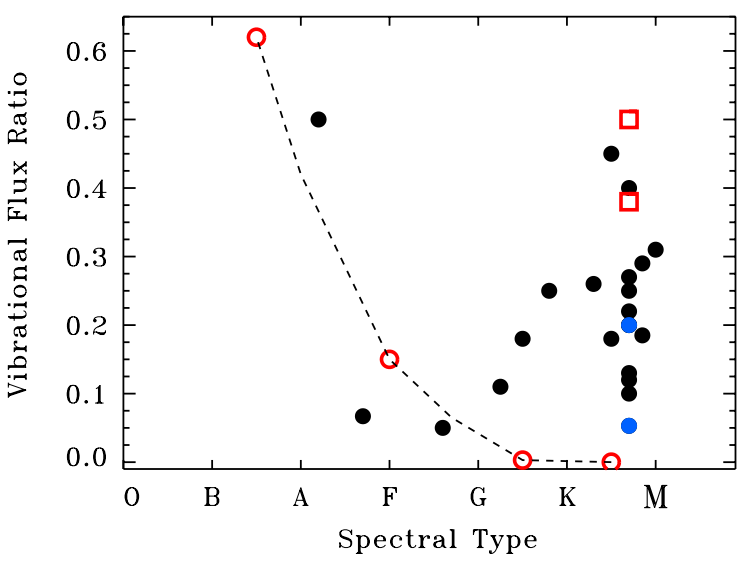

Figure 14. Vibrational flux ratio $(v=2-1 / v=1-0)$ vs. spectral type compared to the pure fluorescence model for vibrational emission (dashed line). Filled black circles are the observed values, open circles are model results for blackbodies at 4000, 6000, 8000, and $10000 \mathrm{~K}$, and squares are model results using the observed UV spectra of DR Tau and TW Hya while the blue dots are the observed values. The observed increased UV flux over blackbody is clearly essential at late spectral types.

(A color version of this figure is available in the online journal.)

coefficients between individual $A-X$ vibrational levels using the Rydberg-Klein-Rees (RKR) program of Le Roy (2004). Accurate potential curves based on the spectroscopic data of Le Floch (1992) for the $X$ state and Field et al. (1972) for the $A$ state were used together with the $A-X$ transition dipole moment of Gilijamse et al. (2007).

One of the main reasons for the expansion to higher vibrational states was to probe the effects of Ly $\alpha$ emission (1216 $\AA$ ), which dominates the far-ultraviolet emission from classical T Tauri stars (Schindhelm et al. 2012) and can pump the $v=14-0$ line of CO (France et al. 2011). Much of the observed Ly $\alpha$ is absorbed by the ISM before reaching Earth so the flux seen by the disk must be reconstructed from fluoresced $\mathrm{H}_{2}$ lines (Herczeg et al. 2002). However, even with reconstructed Ly $\alpha$ line profiles, the effects of $\operatorname{Ly} \alpha$ are only noticeable at high vibrational levels but are marginal at the $v=2-1$ level that is seen in our sample. Some of this result can be explained by the fact that the oscillator strength for the 14-0 band is more than three orders of magnitude smaller than those for the $1-0,2-0$, and $3-0$ bands which dominate the UV pumping. The other factor is that the downward relaxation in the $X$ band is much faster than the UV excitation, spreading the excess flux through all the lower energy vibrational levels.

Figure 14 presents our model results for the vibrational population fraction of the $v=2 / v=1$ vibrational levels for a pure UV fluorescent pumping model using different blackbody radiation fields. The vibrational population fraction decreases with stellar effective temperature due to the smaller amount of UV available to pump the higher vibrational levels of the $A$ state. The observed vibrational flux ratios are included in Figure 14 and generally show much greater values than would be expected from simple blackbodies, especially for the later spectral types. This is in agreement with the correlation found with accretion luminosity in Figure 13. For the cases of TW Hya and DR Tau, both strongly accreting objects, we have also run models using the observed stellar spectra including the enhanced UV emission (Herczeg et al. 2002; Yang et al. 2012), resulting in vibrational flux ratios due to pure UV pumping of $\sim 0.4-0.5$. The observed flux ratios for these sources are 0.05 and 0.2 , respectively, indicated with blue dots in Figure 14. The difference between models and observations is likely due 
to excess population in the $v=1$ levels from IR pumping or collisional excitation, which results in a lower vibration flux ratio. More detailed modeling and observations of higher energy vibrational lines are needed to disentangle these two excitation processes (e.g., Brittain et al. 2007; Brown et al. 2012; Bast et al. 2011). However, the ubiquity and strength of $v=2-1$ lines in the sample appear consistent with UV fluorescent pumping primarily from accretion luminosity.

\section{DISCUSSION}

\subsection{Where is the CO Gas Located?}

The presence of multiple line profile components and temperatures suggests that separate, physically unrelated gas reservoirs are contributing to the spectra. Such reservoirs could include (1) superheated gas from the inner $(\lesssim 1 \mathrm{AU})$ disk surface (Najita et al. 2003; Blake \& Boogert 2004), (2) emission/ absorption from an extended disk wind or other outflow activity (Pontoppidan et al. 2011a; Herczeg et al. 2011), (3) absorption from the outer disk in an inclined system (Rettig et al. 2006), (4) absorption from another disk around a binary companion (Smith et al. 2009), (5) absorption from a remnant envelope (Boogert et al. 2002a; Herczeg et al. 2011), and (6) absorption from cold foreground molecular gas, entirely unrelated to the young star (Boogert et al. 2002b).

Inner disk. The surface layers of disks have long been thought to be one of the primary producers of fundamental $\mathrm{CO}$ emission (e.g., Najita et al. 2003). The gas in the disk atmosphere has regions with the 100-1000 $\mathrm{K}$ temperature range needed to thermally excite the $v=1$ level of $\mathrm{CO}$ and produce the abundant strong CO $v=1-0$ lines. The line widths at the base are consistent with expected Keplerian rotation in the inner regions of disks (Salyk et al. 2011; Appendix B).

Disks with a pure Keplerian velocity field (no radial motions and $V_{\text {phi }} \propto R^{-1 / 2}$ ) produce a characteristic line profile based on inclination. A double peak arises from inclined disks due to the radial decrease in temperature and resulting decrease in low-velocity $\mathrm{CO}$ emission from the cold outer disk causing a central dip. The double peaked line profiles (category 4) are good examples of this classic Keplerian profile and can be seen in the cases of GQ Lup and RNO 90. The central dip disappears at low inclinations due to the lack of velocity contrast between the inner and outer disk resulting in narrow single peaked lines (category 1), such as TW Hya and HD 135344 B. In these cases, the two peaks can still be separated using spectro-astrometry (Pontoppidan et al. 2008).

The list of sources with clear classical Keplerian line profiles consistent with these models is given in Table 3 and is surprisingly small. As can be seen in Figure 5, the majority of our line profiles are single peaked: our percentage of double peaked sources is only $10 \%(7 / 69)$ with six additional sources (category 6) which may be self-absorbed highly inclined disks. This lack of double peaked profiles has been noted in even the earliest CO surveys (Najita et al. 2003). However, the constraints on Keplerian models are greater with the improved spectral and spatial resolution and much large sample. If the inclinations of the full CRIRES sample are randomly distributed and all line profiles are taken to be Keplerian, these low statistics would imply an inclination of $>70^{\circ}$ before a double peaked line profile would be resolved. For a $1 M_{\odot}$ star at the CRIRES spectral resolution, this would imply some $\mathrm{CO}$ emission from $\sim 10 \mathrm{AU}$ or greater, which is not seen in the two-dimensional spectral traces. For less massive stars, limits on the outer extent are less extreme (e.g., $0.5 M_{\odot}$ corresponds to $\sim 5 \mathrm{AU}$ ). However, no trend is seen with spectral type and uniformly low masses are excluded by the range of measured stellar types. We conclude that the warm surface gas in the inner disks in the majority of our sources has kinematics that are inconsistent with only Keplerian rotation.

Disk winds. Many of the sources with the strongest line to continuum ratios are single peaked with broad wings (category 2), including AS 205 A, DR Tau, and RU Lup. As discussed in Bast et al. (2011), the combination of a narrow single peak and broad wings is incompatible with pure Keplerian profiles without high temperatures in the outer disk. The lack of spatial extent rules out this model. Indeed, Pontoppidan et al. (2011a) use spectroastrometry to show that the spatial distribution of the emission is far more compact and asymmetric than can be explained by such a Keplerian disk. They propose a slow moving disk wind as the source of the central emission, but with the broad line wings still due to Keplerian rotation imparted from the launching region of the disk. The line profiles appear symmetric and centered close to the source radial velocity (within $\sim 5 \mathrm{~km} \mathrm{~s}^{-1}$; see Table 4) constraining the physical characteristics of the wind. Sources with these profiles show higher vibrational CO lines indicating fluorescent excitation by the UV radiation produced by the accretion shocks.

The largest category of emission lines is category 3 consisting of the broad profiles $\left(>200 \mathrm{~km} \mathrm{~s}^{-1}\right)$. These were not included in the Bast et al. (2011) sample due to lower contrast between the line width at $10 \%$ compared to $90 \%$ of peak height and/or lower line to continuum ratios. However, based on the prevalence of these single peaked lines, they may have the same origin in a wind.

Outer disk. Category 6 (emission with broad central absorption) sources have strong absorption lines above $P(10)$ $\left(4.7545 \mu \mathrm{m}, E_{\text {upper }}=3330 \mathrm{~K}\right)$ and likely arise from the surface layers of the outer disk. These absorption lines commonly occur in conjunction with disk emission lines although the emission can be difficult to categorize due to the absorption in the line center. Examples of warm CO absorption occur in CW Tau, SR 24, and T Tau S. Temperatures range from 100 to $300 \mathrm{~K}$ (Section 4). At near edge-on inclinations, the outer disk atmosphere could absorb $\mathrm{CO}$ as has been suggested in the case of $\mathrm{T}$ Tau S (Rettig et al. 2006). In the case of binaries, it is possible that the absorption can arise from looking at the star through the companion's disk as may be the case in the VV CrA system where VV CrA N would be seen through the VV CrA S disk (Smith et al. 2009).

Outflows. Molecular outflows and winds moving toward us in our line of sight result in absorption lines with broad blueshifted wings seen in category 7 sources. The gas producing the lines is warm (up to $1000 \mathrm{~K}$ ), likely originating from currently shocked gas rather than entrained outflow gas. The lines are blueshifted due to the disk blocking the redshifted component and its position behind the illuminating star. The wings are not always smooth, indicating clumps in the wind as in the case of DG Tau (shown in Figure 3). LLN 19 has episodic outflows (Thi et al. 2010). VV CrA N also has outflow components which disappear between 2007 April and August. The profiles are similar to those found for the embedded sources in this CRIRES sample highlighted in Herczeg et al. (2011).

The category 8 sources with blue emission and red absorption are probably from a combination of outflow in emission and residual envelope in absorption. Both the emission and 
absorption have similar warm temperatures of a few hundred $\mathrm{K}$. These lines are the most asymmetric of the categories. The warm absorption is close to the cloud velocity and the emission could then be from an outflow. In the case of $\mathrm{T} \mathrm{CrA}$, which is known to drive a jet (Wang et al. 2004), the emission component is spatially extended definitively indicating an origin in an outflow.

Foreground molecular clouds. Many of the CRIRES sources are toward high extinction regions of nearby star forming clouds. $\mathrm{CO}$ gas should be present in the foreground molecular cloud with low turbulent broadening and cold temperatures $(\sim 10-100 \mathrm{~K})$. We attribute the category 5 narrow unresolved absorption lines which appear only at low $J$ levels to this foreground material. This combination of cold temperatures and low densities is confirmed by rotational diagrams (see Section 4). These lines are very common throughout the sample with a detection rate of $38 \%$. The high spectral resolution of CRIRES is a strong advantage for complex line profiles because the foreground lines can be readily separated from emission components and other dips. The binary pairs, such as DoAr 24E, also show the clumpiness of foreground material with even cold absorption components varying between binary pairs on spatial scales of a few 100 AU.

\subsection{Temperature Structure of the Inner Disk Region}

Typical temperatures of the ${ }^{13} \mathrm{CO}$ lines appear lower $(<500 \mathrm{~K})$ than has generally been derived from likely optically thick ${ }^{12} \mathrm{CO}$ lines $(500-1000 \mathrm{~K}) .{ }^{13} \mathrm{CO}$ is close to being optically thin and thus may be able to probe deeper into the disk's vertical structure than ${ }^{12} \mathrm{CO}$. The lower temperatures indicate that the ${ }^{13} \mathrm{CO}$ lines are dominated by emission from either farther out or deeper vertically in the disk. As discussed in Sections 4.1.1 and 4.1.2, both UV and IR pumping may contribute to the line fluxes, but these processes should not change the inferred rotational temperatures significantly.

Line widths systematically differ between ${ }^{12} \mathrm{CO} v=1-0$, $v=2-1$ and ${ }^{13} \mathrm{CO}$, indicating that the lines do not arise from identical locations in the disk. For each disk, the CO $v=2-1$ line profiles are generally broader than $v=1-0$ while the ${ }^{13} \mathrm{CO}$ lines are generally narrower than ${ }^{12} \mathrm{CO} v=1-0$ (Figure 15). This trend was noted by Bast et al. (2011) in their broad single peaked sources but appears more generally throughout the sample. The change in average width between the ${ }^{12} \mathrm{CO}$ and ${ }^{13} \mathrm{CO}$ lines corresponds to a change in radius by a factor of $\sim 4$, assuming Keplerian rotation. If the entire temperature difference between the isotopes is assumed to come from a radial difference, this would imply a temperature profile $T \propto R^{p}$ where $p$ is between -0.5 and -1.5 .

We can also compare our temperature and thermal column density measurements with current chemical models. Many thermo-chemical models of inner disk regions exist and they typically have gas kinetic temperatures of $1000 \mathrm{~K}$ or larger in the layer where $\mathrm{CO}$ becomes abundant (e.g., Gorti \& Hollenbach 2008; Gorti et al. 2011; Glassgold et al. 2009; Woitke et al. 2009; Najita et al. 2011; Walsh et al. 2012; Bruderer et al. 2012). Although there is a large spread in the model results, these temperatures are broadly consistent with those measured for ${ }^{12} \mathrm{CO}$.

Woitke et al. (2009, their Figure 14) provide gas temperatures weighted by the $\mathrm{CO}$ abundance as functions of $\mathrm{CO}$ column density for different disk radii. Their model indicates that the low $J$ CO rovibrational lines become optically thick at $1 \mathrm{AU}$ for a column density of $10^{15} \mathrm{~cm}^{-2}$, at which point the

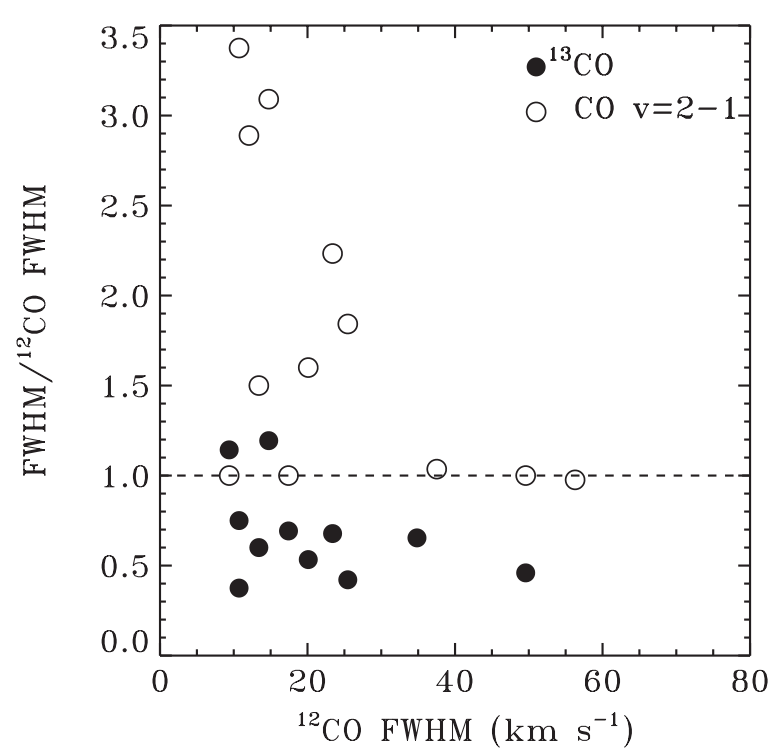

Figure 15. ${ }^{13} \mathrm{CO} \mathrm{FWHM} /{ }^{12} \mathrm{CO}$ FWHM (dots) and ${ }^{12} \mathrm{CO} v=2-1 \mathrm{FWHM} /$ ${ }^{12} \mathrm{CO} v=1-0$ FWHM (open circles) vs. FWHM of the $v=1-0$ lines. The ${ }^{12} \mathrm{CO} v=2-1$ line widths range from similar to the $v=1-0$ lines to much broader. The ${ }^{13} \mathrm{CO}$ line widths are generally down by a factor of $\sim 2$. The two sources with similar FWHM are SR 21, where all emission lines appear to arise from the same region, and $\mathrm{S} \mathrm{CrA} \mathrm{S}$, which has two distinct components in ${ }^{12} \mathrm{CO}$ with the cooler ${ }^{12} \mathrm{CO}$ component having the same line shape as the ${ }^{13} \mathrm{CO}$.

$\mathrm{CO}$ weighted temperature is $600 \mathrm{~K}$. Rarer isotopes, such as ${ }^{13} \mathrm{CO}$, are sensitive to larger column densities before becoming optically thick and thus probe lower temperatures. Our CRIRES ${ }^{13} \mathrm{CO}$ measurements follow the predicted relation between column density and gas temperature in their Figure 14 but lie above the $1 \mathrm{AU}$ contour by a factor of $\sim 3 \times 10^{3}$ in column density. This may be further indication that the ${ }^{13} \mathrm{CO}$ lines are not just thermally excited and thus the column densities are overestimated.

Gorti et al. (2011, their Figure 8) model the CO rovibrational lines in the transitional TW Hya disk and find that inner disk masses between $10^{-4}$ to $10^{-6} M_{\odot}$ fit the rovibrational lines well to within a factor of two. Most of the model emission is dominated by the warm surface layers rather than deeper material. This amount of inner disk gas is similar to those derived from our ${ }^{13} \mathrm{CO}$ data (Table 5).

\subsection{Effects of Stellar Mass on CO}

The fundamental band of $\mathrm{CO}$ from Herbig AeBe stars has been studied extensively (Najita et al. 2003; Blake \& Boogert 2004; Brittain et al. 2007; van der Plas et al. 2009). One of the most obvious differences is the prevalence of high vibration lines in Herbig stars with transitions up to $v=8-7$ being seen (Brittain et al. 2009). In comparison, the T Tauri stars in our sample show vibrationally excited emission generally only to $v=2-1$, with a significant fraction showing no vibrationally excited lines at all. This is probably due to the weaker UV fields emitted from T Tauri stars as is clearly predicted from models (Figure 14).

A comparison of the widths of the line bases (see Appendix B) reveals that Herbig stars tend to have narrower lines (Figure 16). This indicates that the inner edge of the gas is further out in the disk compared to $\mathrm{T}$ Tauri stars. As our sample consists mainly of later type stars, additional Herbig stars from Brittain et al. (2007, 2009) and van der Plas et al. (2009) are included. Our few Herbig stars show similar line widths, indicating that 


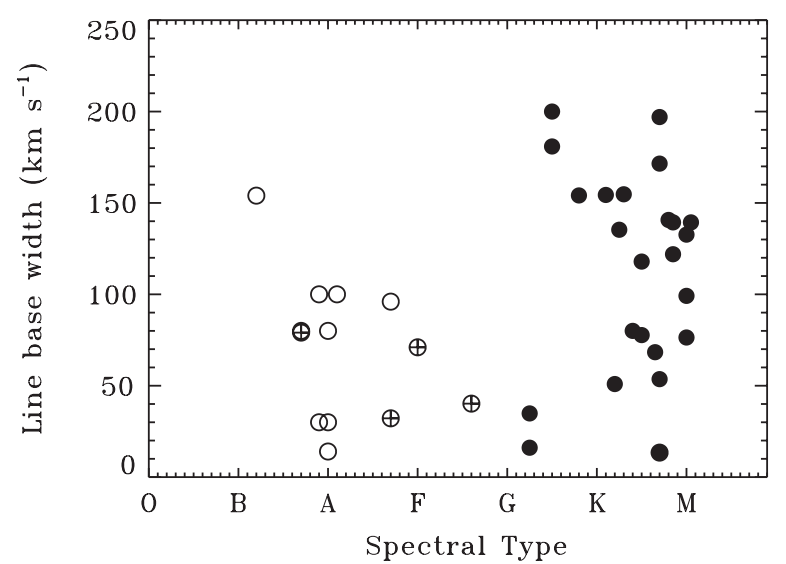

Figure 16. Plot of the line width at base vs. spectral type. This figure shows that earlier type stars tend to have narrower line widths. Our CRIRES sample (filled circles for late-type stars, crossed circles for early-type) consists mainly of later type stars. Additional Herbig stars from Brittain et al. $(2007,2009)$ and van der Plas et al. (2009) are included as open circles.

this is unlikely to be due to any differences in methodology. A Kolmogorov-Smirnov test places the probability at $99 \%$ that the samples have different underlying distributions.

For a given temperature, the radial location will be further out in a Herbig disk than in a T Tauri disk due to the larger luminosities of Herbig stars (Salyk et al. 2011). As the CO lines are sensitive to only a certain range of gas temperatures, the lines would be expected to arise from farther out in Herbig disks. While this explains the general trend that we see, it does not explain the prevalence of Herbig stars with large (>10 AU) gas holes (IRS 48 in our sample, Brown et al. 2012; see also Goto et al. 2006; van der Plas et al. 2009; Brittain et al. 2009). Some additional effect, e.g., photodissociation of $\mathrm{CO}$, is needed to explain these holes.

\subsection{Evolutionary Changes in $\mathrm{CO}$}

Complexity in line profiles generally decreases at each evolutionary stage. Transition disks thus show the most straightfor-

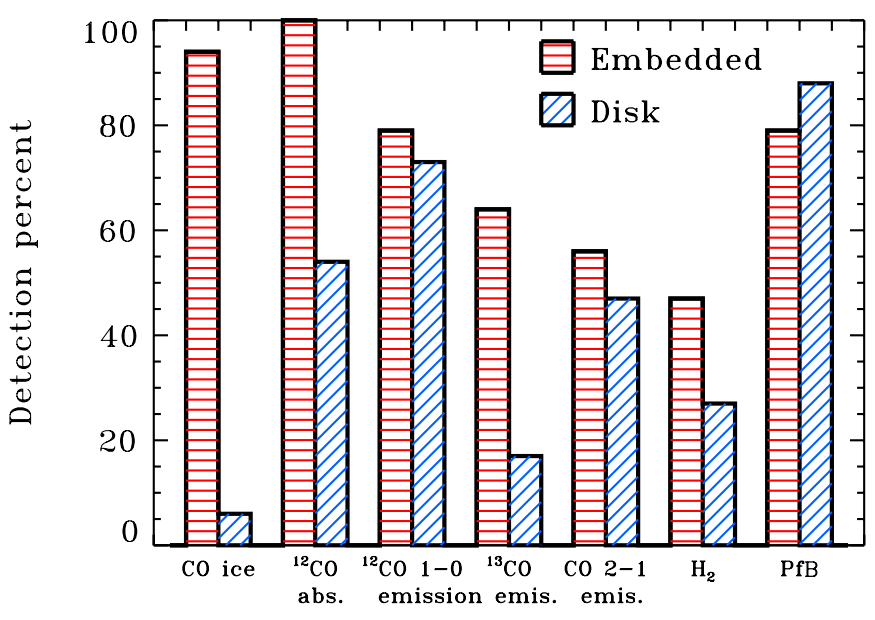

Line

Figure 18. Comparison of the detection percentages of different lines in the embedded (class I) and disk (class II) samples.

(A color version of this figure is available in the online journal.)

ward profiles and embedded class I sources the most kinematically complex.

Class I to Class II. The 18 class I embedded objects discussed by Herczeg et al. (2011) show some systematic differences from the 69 disk sources. The first is that the $\mathrm{CO}$ ice band seen prominently in class I sources (Pontoppidan et al. 2003) disappears from the spectra of the disks due to the disappearance of the cold envelope (Figures 17 and 18). There are only a few exceptions of class II disks with ice bands, with the ice most likely arising in dense foreground gas. Disk winds/outflows as reflected in blue absorption wings are seen in both classes. However, these sources are less common in the disk sample and, when seen, the maximum velocities are smaller (generally $<20 \mathrm{~km} \mathrm{~s}^{-1}$ as opposed to $50-100 \mathrm{~km} \mathrm{~s}^{-1}$ ).

The number of sources with gas phase absorption lines decreases from $100 \%$ to $\sim 60 \%$ as sources evolve from the embedded class I to the class II phase (Figure 18). A comparison

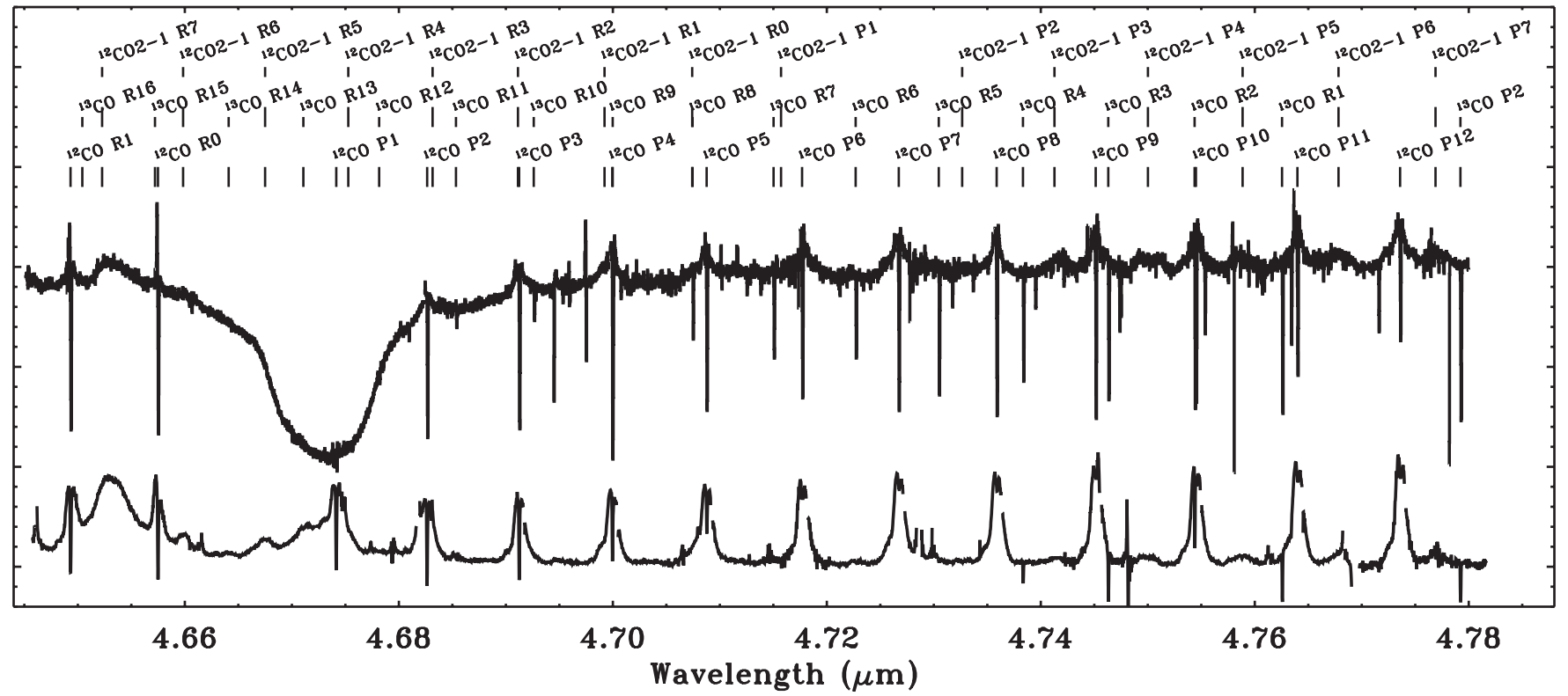

Figure 17. Comparison of the spectra from the class I source IRS 43 (top) and the class II disk RNO 90 (bottom). The deep ice feature at $4.67 \mu \mathrm{m}$ is only present in the class I source. RNO 90 has absorption lines only in the lowest $J{ }^{12} \mathrm{CO}$ lines from cold foreground gas. Most of the differences between the two spectra can be attributed to the disappearance of the envelope. 


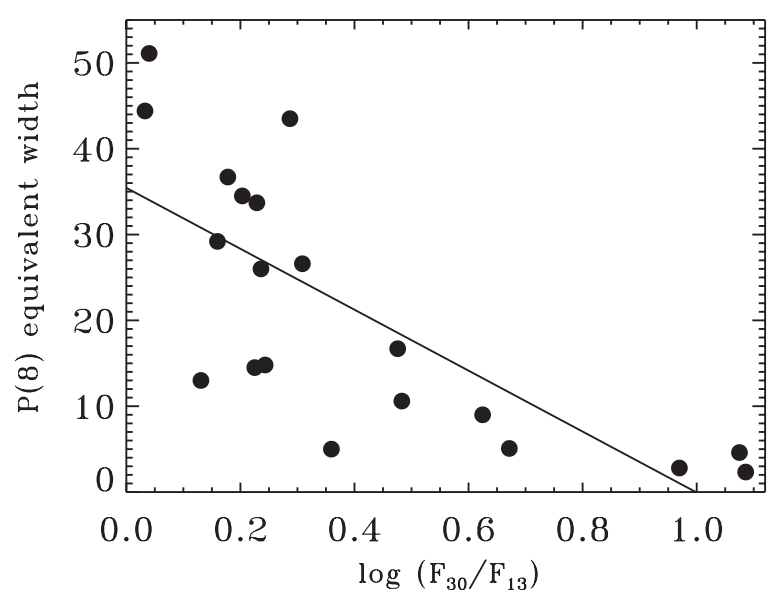

Figure 19. A trend is seen for declining SEDs, as seen in the $13-30 \mu \mathrm{m}$ flux ratios, to have larger $\mathrm{CO} P(8)$ equivalent widths. The flux ratios are taken from Brown et al. (2007), Furlan et al. (2009), and the Spitzer archive. The correlation has a Pearson's correlation coefficient of -0.75 , indicating a strong correlation.

of the absorption line temperatures reveals that the class II sources can show very low temperature $(<50 \mathrm{~K})$ components (Figure 7). However, both class I and class II sources can have warm absorption lines. The inner regions of class I envelopes probably result in higher average temperatures along the line of sight while class II sources may have absorption lines arising solely from cold foreground material. The few class II sources with hundred $\mathrm{K}$ absorption lines likely have an inclination angle such that absorption arises through the surface layers in the outer disk.
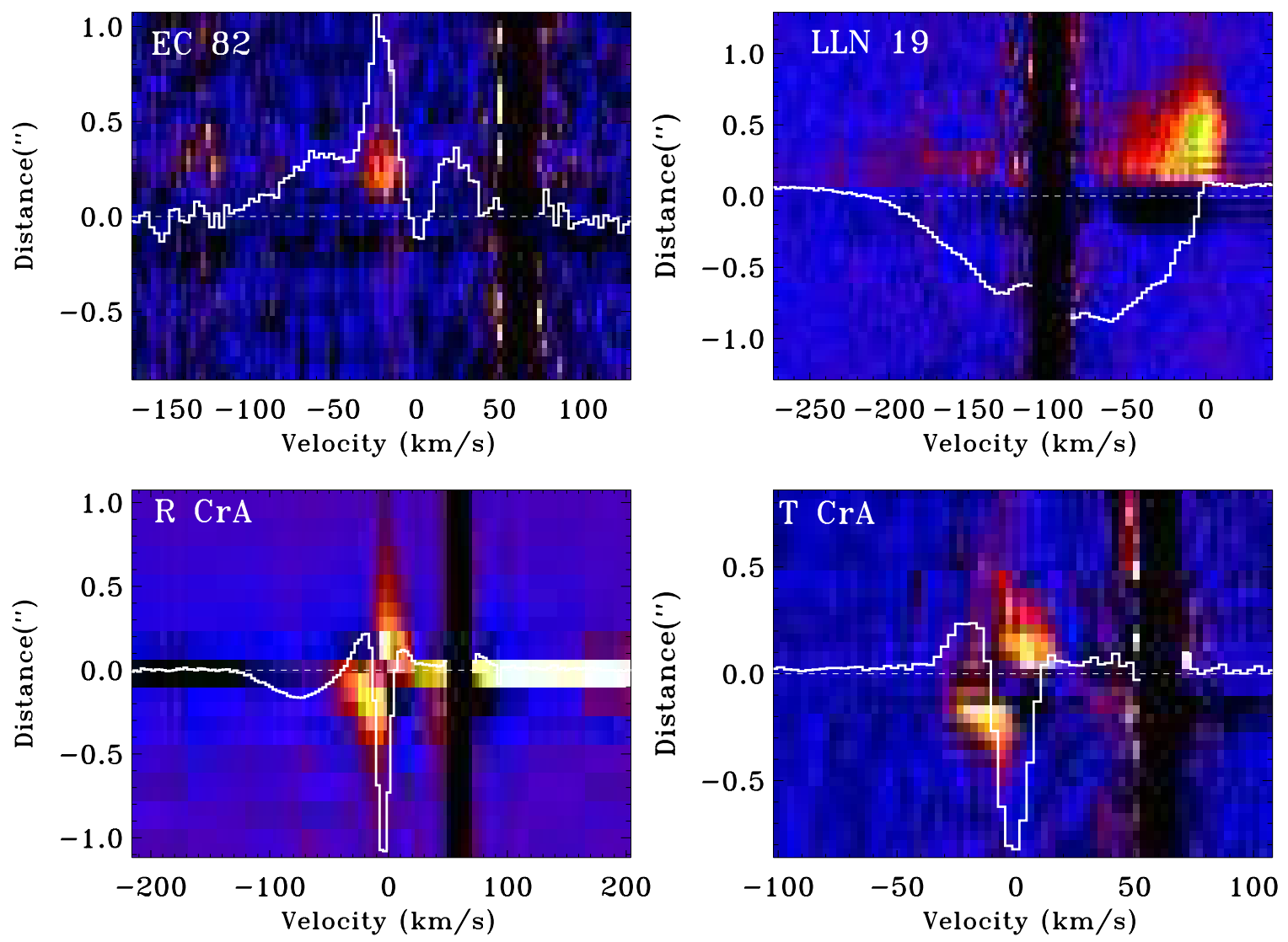

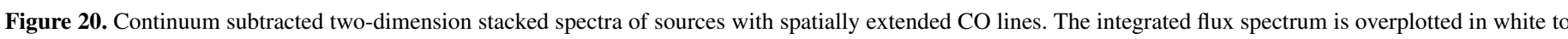
show which components of the line profile are extended. EC 82 and T CrA are at P.A. 90, while R Cra and LLN 19 are at P.A. 0.

(A color version of this figure is available in the online journal.) 


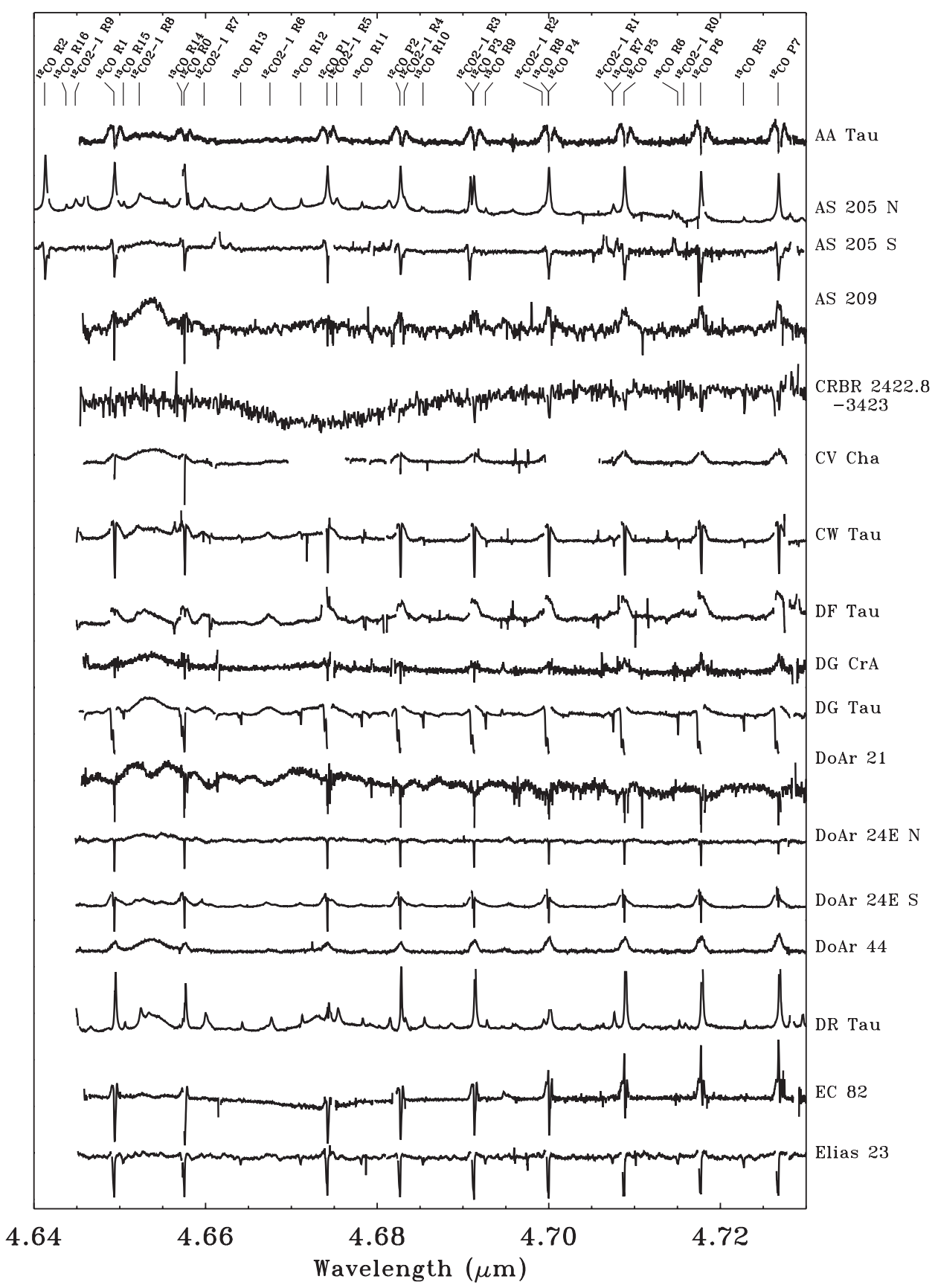

Figure 21. Spectra of the entire CRIRES disk sample in alphabetical order.

(An extended version of this figure is available in the online journal.)

numbers likely indicate strong settling while extremely high numbers mark transitional disks with inner gaps and holes (see Figure 19). A trend is seen with the lowest $n_{13-30}$ sources having the strongest $P(8)$ equivalent widths and the highest $n_{13-30}$ sources having weaker $P(8)$ equivalent widths. This correlation is not reflected in the fluxes, indicating that the cause lies in the interplay between dust continuum and gas lines. This may be an optical depth effect where more $\mathrm{CO}$ resides above the dust $\tau=1$ surface in the settled disks leading to strong CO lines.

\section{CONCLUSIONS}

CO fundamental lines are commonly seen from the circumstellar environments of young stars and provide information on molecular gas in the planet-forming region in disks that ALMA will not be able to probe. Our extensive collection of high spectral resolution CRIRES observations reveal complex environments with signatures of disks, envelopes, foreground molecular clouds, winds, and outflows in diverse combinations.

Young disks display a variety of complex line profiles with simple double-peaked Keplerian disk profiles being surprisingly rare. Most line profiles have wings consistent with Keplerian rotation in the inner disk close to the dust sublimation radius. However, the majority of the emission lines have excess flux at the line center, which in a disk model requires emission from large radii. In most cases, there is no evidence of spatial extent in the $\mathrm{CO}$ lines, ruling out standard Keplerian disk models. This trend, plus a tendency for slight excess emission on the blue side 
of the lines, leads us to conclude that slow disks winds may be common from young stars and traceable through CO lines.

The high spectral resolution and sensitivity of CRIRES allow a more comprehensive overview of emission lines from weaker isotopologues. ${ }^{13} \mathrm{CO}$ lines suffer less confusion from optical depth effects than ${ }^{12} \mathrm{CO}$ lines and thus provide better constraints on the gas properties. The ${ }^{13} \mathrm{CO}$ lines are detected with much greater frequency than in previous studies and have lower excitation temperatures, indicating an origin farther out radially or deeper within the vertical structure. The ${ }^{13} \mathrm{CO}$ line widths are generally narrower than those of ${ }^{12} \mathrm{CO}$, indicating dominance by slower velocity gas arising from larger radii. The ${ }^{13} \mathrm{CO}$ line strengths for the objects with the coldest gas imply large column densities/emitting areas if only collisional excitation is assumed. IR resonant scattering may contribute to the line fluxes, as has been proposed for Herbig $\mathrm{Ae} / \mathrm{Be}$ stars, so that the inferred column densities are upper limits. The derived temperatures are roughly consistent with current thermo/chemical models of the inner disk.

Non-thermal excitation is clearly reflected in the CO lines, hinting at the complex photoprocesses at work in the molecular layer. Vibrational emission from thermally inaccessible energy levels is detected in about $50 \%$ of the sources. This emission is correlated with accretion luminosity indicating that the UV radiation arising from accretion drives the fluorescent pumping, particularly in late-type stars where the stellar UV continuum is weak.

Finally, we examine the large sample for trends between different types of sources. The change from class I embedded protostar to class II disk produces dramatic changes in the $\mathrm{CO}$ spectra, as the removal of the protostellar envelope results in the disappearance of the strong absorption lines and ice feature characteristic of class I spectra. However, the emission lines from class I and II sources, both arising from close to the star, are similar in detection frequency, excitation and line shape, indicating that disk characteristics are established early. The beginning of disk dispersal seen in transition disks also brings changes to the $\mathrm{CO}$ spectra. Transition disk $\mathrm{CO}$ lines are generally Keplerian with a tendency for narrow line widths indicating gas at larger radii. Herbig Ae/Be stars also typically have narrower lines, but in this case, it is likely due to an increase in the dust sublimation radius and the disk region with temperatures suitable for detecting rovibrational $\mathrm{CO}$.

We have highlighted general trends from the large sample to provide an overview of $\mathrm{CO}$ gas within young systems. However, the individual spectra are all unique, reflecting physical differences among the stars, disks, and surrounding environments. We hope that future detailed modeling will be able to exploit fully all the information contained in these rich $\mathrm{CO}$ spectra.

The authors thank Colette Salyk, Jeanette Bast, Wing-Fai Thi, Bill Dent, and Kevin France for discussions and contributions to the program. We also thank the anonymous referee for comments which improved this paper. This work is based on observations collected at the European Southern Observatory Very Large Telescope under program ID 179.C-0151. J.M.B. acknowledges the Smithsonian Astrophysical Observatory for support from a SMA fellowship. Support for K.M.P. was provided by NASA through Hubble Fellowship grant no. 01201.01 awarded by the Space Telescope Science Institute, which is operated by the Association of Universities for Research in Astronomy, Inc., for NASA, under contract NAS 5-26555. Astrochemistry at Leiden is supported by a Spinoza grant from
Table 7

Line Widths

\begin{tabular}{|c|c|c|c|c|}
\hline Source & $\begin{array}{l}\text { Width } 3 \times \text { Noise } \\
\quad\left(\mathrm{km} \mathrm{s}^{-1}\right)\end{array}$ & $\begin{array}{c}\text { Width } 5 \% \text { Peak } \\
\qquad\left(\mathrm{km} \mathrm{s}^{-1}\right)\end{array}$ & $\begin{array}{l}R_{\text {in }} \sin ^{2} i \\
\quad(\mathrm{AU})\end{array}$ & $\begin{array}{c}\text { Inclination } \\
\left({ }^{\circ}\right)\end{array}$ \\
\hline AA Tau & 172 & 172 & 0.060 & 75 \\
\hline AS $205 \mathrm{~N}$ & 103 & 78 & 0.29 & 25 \\
\hline AS 209 & 165 & 90 & 0.22 & \\
\hline CV Cha & 151 & 154 & 0.15 & \\
\hline CW Tau & 142 & 142 & 0.088 & \\
\hline DF Tau & 102 & 118 & 0.13 & \\
\hline DG CrA & 200 & 200 & 0.089 & \\
\hline DG Tau & 142 & 155 & 0.073 & \\
\hline DoAr 24E S & 139 & 135 & 0.097 & \\
\hline DoAr 44 & 90 & 102 & 0.17 & \\
\hline DR Tau & 62 & 54 & 0.62 & 37 \\
\hline EC 82 & 74 & 76 & 0.30 & \\
\hline EX Lup apr & 173 & 250 & 0.028 & \\
\hline EX Lup aug & 134 & 150 & 0.079 & \\
\hline FZ Tau & 50 & 105 & 0.16 & \\
\hline GQ Lup & 139 & 139 & 0.091 & \\
\hline Haro 1-4 A & 64 & 68 & 0.38 & \\
\hline HD $135344 \mathrm{~B}$ & 27 & 32 & 3.43 & 10 \\
\hline HD 142527 & 35 & 40 & 2.20 & \\
\hline HD $144432 \mathrm{~S}$ & 51 & 64 & 2.57 & 48 \\
\hline IRS 48 & 17 & 17 & 37.4 & \\
\hline IRS 51 & 66 & 109 & 0.15 & \\
\hline LkHa 330 & 34 & 35 & 2.92 & 10 \\
\hline RNO 90 & 186 & 181 & 0.11 & \\
\hline RU Lup & 135 & 122 & 0.12 & \\
\hline RY Lup & 51 & 146 & 0.083 & 85 \\
\hline S CrA N & 101 & 99 & 0.36 & \\
\hline S CrA S & 166 & 133 & 0.10 & \\
\hline Serp 64 & 99 & 123 & 0.11 & \\
\hline SR 9 A & 44 & 141 & 0.090 & \\
\hline SR 21 & 13 & 16 & 13.7 & 20 \\
\hline SR $24 \mathrm{~A}$ & 122 & 145 & 0.085 & \\
\hline SR $24 \mathrm{~B} / \mathrm{C}$ & 54 & 83 & 0.26 & \\
\hline T CrA & 39 & 71 & 0.70 & \\
\hline T Tau N & 83 & 146 & 0.17 & \\
\hline T Tau S & 62 & 95 & 0.39 & \\
\hline TW Cha & 67 & 132 & 0.10 & \\
\hline TW Hya & 12 & 13 & 9.9 & 4 \\
\hline VSSG 1 & 118 & 117 & 1.31 & 53 \\
\hline VV CrA S & 60 & 102 & 0.17 & \\
\hline VV Ser & 74 & 79 & 2.84 & \\
\hline VW Cha A & 98 & 139 & 0.091 & \\
\hline VZ Cha & 70 & 99 & 0.18 & \\
\hline Wa Oph 6 & 114 & 197 & 0.046 & 39 \\
\hline WX Cha & 127 & 332 & 0.016 & \\
\hline
\end{tabular}

the Netherlands Organization for Scientific Research (NWO), by NWO grant 614.000 .605 , and by the Netherlands Research School for Astronomy (NOVA).

\section{APPENDIX A}

\section{SOURCES WITH EXTENDED EMISSION}

Figure 20 presents the emission of the three additional sources in our disk sample for the lines can be directly imaged: EC 82, R CrA, and T CrA. LLN 19, a massive embedded object, also shows extended emission. As found previously for the embedded sources GSS 30 and IRS 43 by Herczeg et al. (2011), most of the visually extended sources appear to be outflows with the spatially extended emission occurring on only one side of the spectral trace, probably in the outflow cavity wall (see Figure 20). 
Table 8

CRIRES Observations of Embedded Young Stars

\begin{tabular}{|c|c|c|}
\hline Source & $\begin{array}{l}\text { Wavelength } \\
\quad(\mathrm{nm})\end{array}$ & Date of Observation $^{\mathrm{a}}$ \\
\hline CrA IRS 2 & $4716,4730,4833,4929.3$ & 2007 Apr 25 \\
\hline EC $90 \mathrm{~N} \& \mathrm{~S}$ & $4716,4730,4929.3,4946.2$ & 2007 Apr 23 \\
\hline Elias 29 & $4716,4730,4868$ & 2008 Aug 8 \\
\hline Elias 32 & $4716,4730,4833 ; 4716 ; 4730$ & 2008 May 2; 2008 Aug 8; 9 \\
\hline GSS 30 & 4716,$4730 ; 5115$ & 2010 Mar 4; 2008 Aug 4 \\
\hline IRS 43 & $4716,4730,4868 ; 4831$ & 2008 Aug 5; 2008 Aug 6 \\
\hline IRS 44 E \& W & $4716 ; 4730,4833 ; 4946.2 ; 4868 ; 4716,4730$ & 2008 Apr 26; 29; 30; 2008 Aug 5; 6 \\
\hline IRS 63 & $4716,4730,4833,4929.3 ; 4716,4730,4868$ & 2007 Apr 25; 2008 Aug 5 \\
\hline L1551 & 4716,4730 & 2007 Oct 16 \\
\hline L1689 IRS 5 & 4716,$4730 ; 4716,4730,4800,5115$ & 2010 Mar 10; 2010 Mar 15 \\
\hline LLN 8 & 4716,4730 & 2007 Oct 11 \\
\hline LLN 17 & 4716,$4730 ; 4716,4730,4868$ & 2007 Oct $11 ; 2007$ Oct 13 \\
\hline RE 50 & $4716,4730,4868 ; 4770$ & 2007 Oct 11, 2007 Oct 17 \\
\hline RNO 91 & $\begin{array}{l}4716,4730,4929.3,4946.2 ; 4770,4779.5 ; \\
4716,4730 ; 4800,4820\end{array}$ & $\begin{array}{l}2007 \text { Apr 23; Apr 24; } \\
2010 \text { Mar 19; } 2010 \text { Mar } 9\end{array}$ \\
\hline TMC 1A & 4716,$4730 ; 4800,4946.2$ & 2010 Feb 10; 2010 Feb 3 \\
\hline WL 12 & 4716,$4730 ; 4833$ & 2007 Sep 1; 2007 Sep 3 \\
\hline WLY 2-42 & $4716,4730,4833$ & 2008 Apr 26 \\
\hline
\end{tabular}

Notes. ${ }^{\text {a }}$ Equivalent to Table 1 for the embedded sample.

b Source was incorrectly labelled as WL 6 in Herczeg et al. (2011).

Evidence for outflows is also seen in broad absorption lines for these and other sources (Figures 3 and 4; see also Thi et al. 2010; Herczeg et al. 2011). In contrast with the slow molecular winds analyzed through spectro-astrometry (Pontoppidan et al. 2011a), the outflows show large blueshifts up to a few $100 \mathrm{~km} \mathrm{~s}^{-1}$ from source velocity.

\section{APPENDIX B}

\section{INNER RADII}

The gas closest to the star rotates most rapidly, producing the wings of the line profile. Assuming that the CO lines arise from such a disk, the innermost extent of the $\mathrm{CO}$ can be measured based on the width of the line base. Two different methods of measuring the width of the stacked line profiles were used to overcome this problem (Table 7). In the first, the width was measured between the points where the line rose above three times the continuum noise. In the second, the width was measured at $5 \%$ of the peak flux values. These two measurements are often similar for our typical noise levels. When the continuum noise is low the first method provides a better grasp of the actual extent of the gas. Inner radii were determined by stacking clean lines to decrease continuum noise.

The derived number is actually in terms of $v \sin i$ and thus the resulting radius is dependent on the inclination and stellar mass. The corresponding $R \sin ^{2} i$ was calculated assuming Keplerian rotation and a typical stellar mass for the spectral type $(\mathrm{O}, \mathrm{B}$ : $5 M_{\odot}$; A: $3 M_{\odot}$; F, G: $1 M_{\odot}$; and K, M: $0.5 M_{\odot}$ ). The resulting numbers are highly dependent on inclination particularly for low inclination sources. For example, TW Hya, which has a known inclination of $4^{\circ}-7^{\circ}$ (Pontoppidan et al. 2008; Qi et al. 2004), has an $R \sin ^{2} i$ of $9.9 \mathrm{AU}$ but an actual inner radius of less than 0.048-0.15 AU. Also, any disk turbulence will broaden the line, resulting in a smaller estimated inner radius than is actually the case. The disk turbulence is likely to be small compared to the line widths of $>50 \mathrm{~km} \mathrm{~s}^{-1}$.
The distribution of $v \sin i$ for the T Tauri stars is flat within the errors as expected if inclination is the dominant effect. The range of values is consistent with Keplerian emission from the inner disk close to the dust sublimation radii as measured by infrared interferometry (e.g., Akeson et al. 2005). The smallest $v \sin i$ values belong to the single peaked Keplerian sources, which in our sample are mainly transition disks with known low inclinations. The largest values tend to belong to the double peaked Keplerian sources.

\section{APPENDIX C}

\section{GALLERY OF SPECTRA}

Spectra of the entire CRIRES sample are presented in Figure 21. Figure 21 shows $4.64-4.63 \mu \mathrm{m}\left[{ }^{12} \mathrm{CO} R(0)-P(7)\right]$ for all the disks in alphabetical order (Figure 21: A-EL, EXIR, IR-SY, SY-Z). Eight disk sources, mainly in Chamaeleon, were observed only in the longer wavelength settings. These sources are denoted with ${ }^{*}$ in the first part of Figure 21 and spectra covering $4.77-4.85 \mu\left[{ }^{12} \mathrm{CO} P(12)-P(18)\right]$ are shown in a separate panel. The 22 embedded sources, including higher mass sources not covered in Herczeg et al. (2011), are shown at the end of Figure 21. Table 8 presents the observation log for the entire embedded source sample.

\section{REFERENCES}

Aikawa, Y., van Zadelhoff, G. J., van Dishoeck, E. F., \& Herbst, E. 2002, A\&A, 386,622

Akeson, R. L., Boden, A. F., Monnier, J. D., et al. 2005, ApJ, 635, 1173

Alcala, J. M., Covino, E., Franchini, M., et al. 1993, A\&A, 272, 225

Armitage, P. J. (ed.) 2010, Astrophysics of Planet Formation (Cambridge: Cambridge Univ. Press)

Armitage, P. J. 2011, ARA\&A, 49, 195

Armitage, P. J., Clarke, C. J., \& Palla, F. 2003, MNRAS, 342, 1139

Bast, J. E., Brown, J. M., Herczeg, G. J., van Dishoeck, E. F., \& Pontoppidan, K. M. 2011, A\&A, 527, A119

Blake, G. A., \& Boogert, A. C. A. 2004, ApJL, 606, L73

Boogert, A. C. A., Hogerheijde, M. R., \& Blake, G. A. 2002a, ApJ, 568, 761 
Boogert, A. C. A., Hogerheijde, M. R., Ceccarelli, C., et al. 2002b, ApJ, 570,708

Brittain, S. D., Najita, J. R., \& Carr, J. S. 2009, ApJ, 702, 85

Brittain, S. D., Simon, T., Najita, J. R., \& Rettig, T. W. 2007, ApJ, 659, 685

Brown, J. M. 2008, PhD thesis, California Institute of Technology

Brown, J. M., Blake, G. A., Dullemond, C. P., et al. 2007, ApJL, 664, L107

Brown, J. M., Herczeg, G. J., Pontoppidan, K. M., \& van Dishoeck, E. F. 2012, ApJ, 744, 116

Bruderer, S., van Dishoeck, E. F., Doty, S. D., \& Herczeg, G. J. 2012, A\&A, 541, A91

Correia, S., Zinnecker, H., Ratzka, T., \& Sterzik, M. F. 2006, A\&A, 459, 909

Dunkin, S. K., Barlow, M. J., \& Ryan, S. G. 1997, MNRAS, 290, 165

Evans, N. J., II, Allen, L. E., Blake, G. A., et al. 2003, PASP, 115, 965

Evans, N. J., II, Dunham, M. M., Jørgensen, J. K., et al. 2009, ApJS, 181, 321

Field, R. W., Tilford, S. G., Howard, R. A., \& Simmons, J. D. 1972, JMoSp, 44, 347

Flaherty, K., Muzerolle, J., Rieke, G., et al. 2012, ApJ, 748, 71

France, K., Schindhelm, E., Burgh, E. B., et al. 2011, ApJ, 734, 31

Furlan, E., Watson, D. M., McClure, M. K., et al. 2009, ApJ, 703, 1964

Gammie, C. F. 1996, ApJ, 457, 355

Gilijamse, J. J., Hoekstra, S., Meek, S. A., et al. 2007, JChPh, 127, 221102

Glassgold, A. E., Meijerink, R., \& Najita, J. R. 2009, ApJ, 701, 142

Gorti, U., \& Hollenbach, D. 2008, ApJ, 683, 287

Gorti, U., Hollenbach, D., Najita, J., \& Pascucci, I. 2011, ApJ, 735, 90

Goto, M., Regály, Z., Dullemond, C. P., et al. 2011, ApJ, 728, 5

Goto, M., Usuda, T., Dullemond, C. P., et al. 2006, ApJ, 652, 758

Greene, T. P., \& Meyer, M. R. 1995, ApJ, 450, 233

Guenther, E. W., Esposito, M., Mundt, R., et al. 2007, A\&A, 467, 1147

Hartmann, L., Megeath, S. T., Allen, L., et al. 2005, ApJ, 629, 881

Herbig, G. H., \& Bell, K. R. 1988, Third Catalog of Emission-line Stars of the Orion Population (Santa Cruz, CA: Lick Observatory), 3

Herczeg, G. J., Brown, J. M., van Dishoeck, E. F., \& Pontoppidan, K. M. 2011, A\&A, 533, A112

Herczeg, G. J., Linsky, J. L., Valenti, J. A., Johns-Krull, C. M., \& Wood, B. E. 2002, ApJ, 572, 310

Hillenbrand, L. A., Strom, S. E., Vrba, F. J., \& Keene, J. 1992, ApJ, 397, 613

Hollenbach, D., Johnstone, D., Lizano, S., \& Shu, F. 1994, ApJ, 428, 654

Kaeufl, H.-U., Ballester, P., Biereichel, P., et al. 2004, Proc. SPIE, 5492, 1218

Kenyon, S. J., \& Hartmann, L. 1995, ApJS, 101, 117

Kessler-Silacci, J., Augereau, J.-C., Dullemond, C. P., et al. 2006, ApJ, 639, 275

Kley, W., \& Nelson, R. P. 2012, ARA\&A, 50, 211

Köhler, R., Neuhäuser, R., Krämer, S., et al. 2008, A\&A, 488, 997

Le Floch, A. 1992, MolPh, 72, 133

Le Roy, R. J. 2004, Chemical Physics Research, Report No. CP-657R

Luhman, K. L., Allen, L. E., Allen, P. R., et al. 2008, ApJ, 675, 1375

Luhman, K. L., Allen, P. R., Espaillat, C., Hartmann, L., \& Calvet, N. 2010, ApJS, 186, 111

Luhman, K. L., Whitney, B. A., Meade, M. R., et al. 2006, ApJ, 647, 1180

Malfait, K., Bogaert, E., \& Waelkens, C. 1998, A\&A, 331, 211

Mandell, A. M., Bast, J., van Dishoeck, E. F., et al. 2012, ApJ, 747, 92

Markwick, A. J., Ilgner, M., Millar, T. J., \& Henning, T. 2002, A\&A, 385, 632

McCabe, C., Ghez, A. M., Prato, L., et al. 2006, ApJ, 636, 932
Melo, C. H. F. 2003, A\&A, 410, 269

Merín, B., Jørgensen, J., Spezzi, L., et al. 2008, ApJS, 177, 551

Muzerolle, J., Flaherty, K., Balog, Z., et al. 2009, ApJL, 704, L15

Muzerolle, J., Hillenbrand, L., Calvet, N., Briceño, C., \& Hartmann, L. 2003, ApJ, 592, 266

Nagasawa, M., Thommes, E. W., Kenyon, S. J., Bromley, B. C., \& Lin, D. N. C. 2007, in Protostars and Planets V, ed. B. Reipurth, D. Jewitt, \& K. Keil (Tucson, AZ: Univ. Arizona Press), 639

Najita, J., Carr, J. S., \& Mathieu, R. D. 2003, ApJ, 589, 931

Najita, J. R., Ádámkovics, M., \& Glassgold, A. E. 2011, ApJ, 743, 147

Oliveira, I., Merín, B., Pontoppidan, K. M., et al. 2009, ApJ, 691, 672

Owen, J. E., Ercolano, B., Clarke, C. J., \& Alexander, R. D. 2010, MNRAS, 401, 1415

Padgett, D. L., Cieza, L., Stapelfeldt, K. R., et al. 2006, ApJ, 645, 1283

Panić, O., Hogerheijde, M. R., Wilner, D., \& Qi, C. 2009, A\&A, 501, 269

Perez-Becker, D., \& Chiang, E. 2011, ApJ, 735, 8

Peterson, D. E., Caratti o Garatti, A., Bourke, T. L., et al. 2011, ApJS, 194, 43

Pontoppidan, K. M., Blake, G. A., \& Smette, A. 2011a, ApJ, 733, 84

Pontoppidan, K. M., Blake, G. A., van Dishoeck, E. F., et al. 2008, ApJ, 684, 1323

Pontoppidan, K. M., Fraser, H. J., Dartois, E., et al. 2003, A\&A, 408, 981

Pontoppidan, K. M., van Dishoeck, E., Blake, G. A., et al. 2011b, Msngr, 143,32

Qi, C., Ho, P. T. P., Wilner, D. J., et al. 2004, ApJL, 616, L11

Raymond, S. N., Quinn, T., \& Lunine, J. I. 2004, Icar, 168, 1

Regály, Z., Sándor, Z., Dullemond, C. P., \& van Boekel, R. 2010, A\&A, 523, A69

Rettig, T., Brittain, S., Simon, T., et al. 2006, ApJ, 646, 342

Salyk, C., Blake, G. A., Boogert, A. C. A., \& Brown, J. M. 2009, ApJ, 699, 330

Salyk, C., Blake, G. A., Boogert, A. C. A., \& Brown, J. M. 2011, ApJ, 743, 112

Salyk, C., Herczeg, G. J., Brown, J. M., et al. 2013, ApJ, 769, 21

Salyk, C., Pontoppidan, K. M., Blake, G. A., et al. 2008, ApJL, 676, L49

Schindhelm, E., France, K., Herczeg, G. J., et al. 2012, ApJL, 756, L23

Setiawan, J., Henning, T., Launhardt, R., et al. 2008, Natur, 451, 38

Smith, R. L., Pontoppidan, K. M., Young, E. D., Morris, M. R., \& van Dishoeck, E. F. 2009, ApJ, 701, 163

Thi, W., van Dishoeck, E. F., Pontoppidan, K. M., \& Dartois, E. 2010, MNRAS, 406, 1409

Torres, C. A. O., Quast, G. R., da Silva, L., et al. 2006, A\&A, 460, 695

van der Plas, G., van den Ancker, M. E., Acke, B., et al. 2009, A\&A, 500, 1137

van Dishoeck, E. F., Dartois, E., Pontoppidan, K. M., et al. 2003, Msngr, 113,49

van Kempen, T. A., van Dishoeck, E. F., Brinch, C., \& Hogerheijde, M. R. 2007, A\&A, 461, 983

Walsh, C., Nomura, H., Millar, T. J., \& Aikawa, Y. 2012, ApJ, 747, 114

Walter, F. M., Herczeg, G., Brown, A., et al. 2003, AJ, 126, 3076

Wang, H., Mundt, R., Henning, T., \& Apai, D. 2004, ApJ, 617, 1191

White, R. J., \& Hillenbrand, L. A. 2004, ApJ, 616, 998

Woitke, P., Kamp, I., \& Thi, W.-F. 2009, A\&A, 501, 383

Woods, P. M., \& Willacy, K. 2009, ApJ, 693, 1360

Yang, H., Herczeg, G. J., Linsky, J. L., et al. 2012, ApJ, 744, 121 